ARTICLE

DOI: $10.1038 / s 41467-017-02045-7$

\title{
Evolution of a flipped pathway creates metabolic innovation in tomato trichomes through BAHD enzyme promiscuity
}

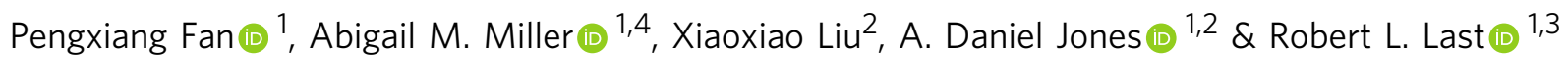

Plants produce hundreds of thousands of structurally diverse specialized metabolites via multistep biosynthetic networks, including compounds of ecological and therapeutic importance. These pathways are restricted to specific plant groups, and are excellent systems for understanding metabolic evolution. Tomato and other plants in the nightshade family synthesize protective acylated sugars in the tip cells of glandular trichomes on stems and leaves. We describe a metabolic innovation in wild tomato species that contributes to acylsucrose structural diversity. A small number of amino acid changes in two acylsucrose acyltransferases alter their acyl acceptor preferences, resulting in reversal of their order of reaction and increased product diversity. This study demonstrates how small numbers of amino acid changes in multiple pathway enzymes can lead to diversification of specialized metabolites in plants. It also highlights the power of a combined genetic, genomic and in vitro biochemical approach to identify the evolutionary mechanisms leading to metabolic novelty.

\footnotetext{
${ }^{1}$ Department of Biochemistry and Molecular Biology, Michigan State University, East Lansing, MI 48824, USA. ${ }^{2}$ Department of Chemistry, Michigan State University, East Lansing, MI 48824, USA. ${ }^{3}$ Department of Plant Biology, Michigan State University, East Lansing, MI 48824, USA. ${ }^{4}$ Present address: Department of Molecular Biology and Genetics, Cornell University, Ithaca, NY 14853, USA. Correspondence and requests for materials should be addressed to R.L.L. (email: lastr@msu.edu)
} 
$\mathrm{P}$ lants produce large numbers of structurally diverse specialized metabolites ${ }^{1}$, with individual classes typically restricted both taxonomically and spatiotemporally $y^{2,3}$. The key families of enzymes involved in producing these metabolites, e.g., cytochrome P450-dependent monooxygenases, terpene synthases, and BAHD acyltransferases, show strong signs of diversification over the course of land plant evolution ${ }^{4}$, giving rise to hundreds of thousands of structurally diverse plant metabolites with roles ranging from pollinator and symbiotic interaction to pathogen and herbivore defense ${ }^{1,5-7}$. These enzymes exhibit signs of rapid evolution compared with those of core metabolism ${ }^{8}$, and a variety of factors are associated with this lability. For example, specialized metabolic enzymes are encoded by multigene families, and genetic redundancy creates the potential for evolution of novel regulation or enzymatic activities with reduced negative impacts on fitness 9 . In addition, the cell and tissue specificity of specialized metabolic pathways is conducive to changes in enzyme activities without causing adverse fitness effects. Finally, enzyme promiscuity-the ability to utilize multiple structurally related substrates-is associated with the ability of specialized metabolic enzymes to evolve rapidly ${ }^{10-13}$. Changes in one enzymatic activity influence its ability to use substrates produced by other enzymes, and in turn generate products that are substrates for other enzymes. Understanding the evolution of pairs or sets of enzymes can contribute to our knowledge of catalysis and inform novel metabolic engineering strategies.

Several characteristics make the acylsucrose biosynthetic network in cultivated tomato (Solanum lycopersicum) and its wild relatives an exemplary system for understanding biosynthetic pathway evolution. Acylsugars are of interest as natural plant pest control agents, with documented direct protection from fungal pathogen $^{14}$ and herbivore ${ }^{14-17}$ attack, and indirect protection through tri-trophic interactions ${ }^{18,19}$. The core network in cultivated tomato is relatively simple, with four glandular trichomeexpressed BAHD (BEAT, AHCT, HCBT, DAT ${ }^{20,21}$ ) class acylsucrose acyltransferases (ASATs) catalyzing consecutive reactions $^{22}$. In recent studies, ASATs from Solanaceae species distantly related to the tomato group of Solanum-Petunia axillaris and Salpiglossis sinuata-were characterized, and these create acylsucroses with structures different from those of wild and cultivated tomatoes ${ }^{23,24}$. In cultivated tomato, the entire acylsugar biosynthetic pathway can function in a single test tube with addition of sucrose and acyl-CoA substrates to the four enzymes, permitting facile characterization ${ }^{22,25}$. Despite the compact pathway structure, these enzymes produce diverse triand tetra-acylated products in cultivated tomato, due in part to the acyl-CoA substrate promiscuity of the second and third enzymes in the pathway ${ }^{22,26-28}$. Study of wild tomato relatives in the Solanum revealed examples of enzyme and product diversification compared with the domesticated species ${ }^{27-30}$. For example, populations of $S$. habrochaites from northern Ecuador lack activity of ASAT4, the last enzyme of the pathway, resulting in loss of pyranose ring $R_{2}$ acetylation ${ }^{29}$. Interspecific differences in ASAT2 and ASAT3 acyl-CoA substrate preference cause acyl chain variation at the pyranose $R_{4}$ and $R_{3^{\prime}}$ positions, respectively ${ }^{22,27}$. Despite these differences, most wild and cultivated tomato acylsucroses contain a single acyl chain on the furanose ring at the sucrose $\mathrm{R}_{3}$ ' position ("-F type" acylsucroses, see Fig. 1a) $)^{27,29}$.

In contrast, $S$. habrochaites and $S$. pennellii accumulate an unusual set of triacylated sucroses decorated only on the sixmember pyranose ring ("-P type" acylsucroses, see Fig. 1a) ${ }^{28,30}$. We found that these species produce ASAT3-P enzyme, which is a variant that uses a monoacylated acylsucrose acceptor substrate, rather than the diacylated substrates used by S. lycopersicum type Sl-ASAT3 activity ${ }^{27}$. However, this work left open the question of a

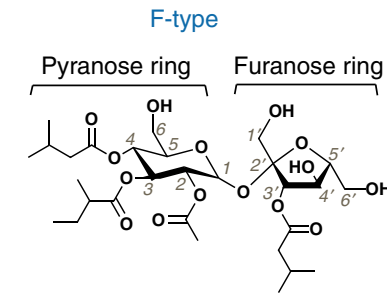

S4:17 (2,5,5,5)-F
S. lycopersicum M82

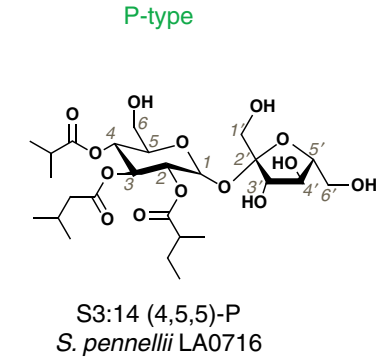

1: TOF MS ES-
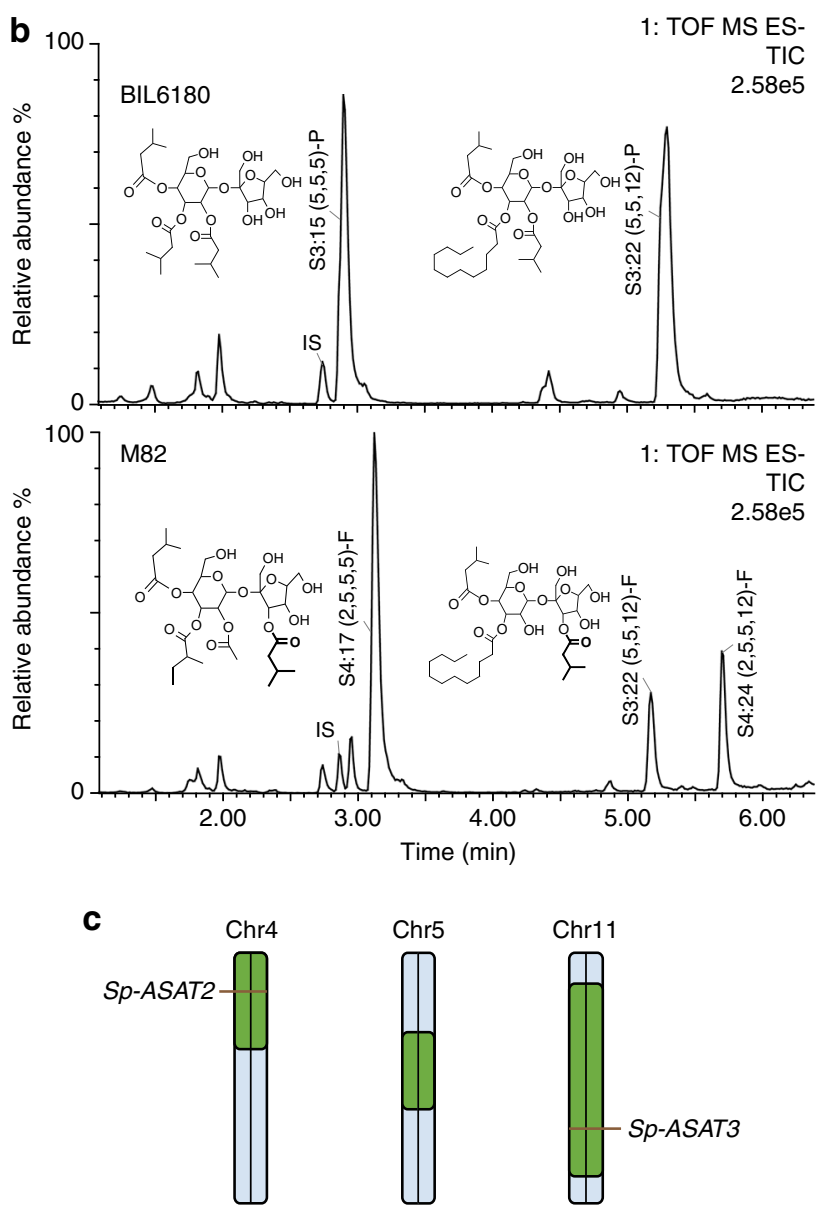

Fig. 1 The recombinant line BIL6180 produces abundant acylsucroses with all acyl chains on the pyranose ring. a Structures of representative F- and Ptype acylsucroses purified from S. lycopersicum M82 and S. pennellii LA0716, respectively. The F-type acylsucrose has acylations on the pyranose and furanose rings, with a single acyl chain at $R_{3^{\prime}}$ position. The P-type acylsucrose has three acyl chains on the pyranose ring, with no furanose ring acylation. b BIL6180 produces abundant P-type acylsucroses, while S. lycopersicum M82 accumulates mainly tri- and tetra- F-type acylsucroses in the trichomes. IS: internal standard. The two total ion chromatograms are scaled to the same intensity, where the major peak (S4:17-F) in M82 has the maximum signal (100\% scale). c BIL6180 has three chromosome regions from S. pennellii (green) introgressed into S. lycopersicum M82 background (blue). The introgressions on chromosomes 4 and 11 include Sp-ASAT2 and Sp-ASAT3, respectively

what enzyme converts the diacylated ASAT3-P product to the final triacylated P-type acylsucrose, which has all three substitutions on the pyranose ring.

In the current study, we address this question using a combination of genetic and transgenic plant approaches along with site- 
directed mutagenesis and in vitro biochemistry. We identify a cultivated tomato line that produces P-type acylsucroses and found that the concerted action of two regions of the S. pennellii LA0716 genome causes this novel phenotype. These regions harbor the $S p$-ASAT2 and Sp-ASAT3 genetic loci, and P-type acylsucrose accumulation results from epistasis between these loci. The combination of these alleles is necessary and sufficient to produce the pyranose ring acylated products in vivo. Biochemical analysis demonstrates that the P-type acylsucroses result from reaction of Sp-ASAT3 followed by Sp-ASAT2, which is the reverse order of action compared with that of the cultivated tomato enzymes. In vitro mutagenesis reveals that a small number of amino acid changes in each enzyme is sufficient to switch the activities between the F- and P- acylsucrose pathways. Investigation of these pathways in other tomato species allows inference of the evolutionary events giving rise to divergence of Fand P-type acylsucroses. This work demonstrates multiple evolutionary mechanisms in biochemical pathway evolution, including gene duplication and loss, amino acid substitution, and how emergence of pathway epistatic interactions restructured a specialized metabolic pathway and led to metabolic product innovation.

\section{Results}

Two $S$. pennellii loci influence acylsucrose acylations. Single chromosomal introgressions from S. pennellii LA0716 cause dramatic changes in the types of specialized metabolites that accumulate in cultivated tomato trichomes ${ }^{22,26,27,30-34}$ and other tissues $^{35}$. The availability of Backcrossed Inbred Lines (BILs), containing multiple $S$. pennellii chromosomal regions in individual $S$. lycopersicum lines ${ }^{33,35,36}$ provides an opportunity to identify epistatic interactions that influence the types of metabolites that accumulate. We analyzed trichome acylsugar metabolites of 257 BILs by liquid chromatography-time of flight mass spectrometry (LC-ToF MS) to look for phenotypes not observed in single chromosomal introgression lines.

One BIL line-referred to as 6180 in this report-was found to produce an acylsugar profile absent from S. lycopersicum M82 or single introgression lines (ILs) previously screened ${ }^{32}$ (Fig. 1b). BIL6180 exclusively accumulated detectable triacylsucroses with all three acylchains on one ring, as revealed by positive mode mass spectrometry (MS) (Supplementary Fig. 1a). These resemble the acylsugars produced in S. pennellii and S. habrochaites ${ }^{27}$ and the minor acylsugar peaks produced in IL4-1 ${ }^{22}$. NMR analysis of two of the major BIL6180 triacylsucrose peaks, namely S3:15 (5, $5,5)$ and $S 3: 22(5,5,12)$ (note that "S" refers to a sucrose backbone, "3:15" indicates three acyl chains with total fifteen carbons and the length of each acyl chain is shown in the parentheses), showed that they were acylated exclusively on the six-membered pyranose ring at the $\mathrm{R}_{2}, \mathrm{R}_{3}$, and $\mathrm{R}_{4}$ positions (Supplementary Fig. 1b). This pattern is in contrast to that of the parent S. lycopersicum, which accumulates F-type acylsucroses with a single acyl chain at the $\mathrm{R}_{3^{\prime}}$ position of the five-membered furanose ring (Fig. 1a). However, it resembles the acylation pattern of the S. pennellii LA0716 acylsucrose S3:19-P $\left(5^{\mathrm{R} 2}, 10^{\mathrm{R} 3}\right.$, $4^{\mathrm{R} 4}$ ) previously resolved by $\mathrm{NMR}^{30}$. Another similarity between the BIL6180 and S. pennellii LA0716 acylsucroses is that both $\mathrm{S} 3: 22-\mathrm{P}\left(5^{\mathrm{R} 2}, 12^{\mathrm{R} 3}, 5^{\mathrm{R} 4}\right)$ and S3:19-P $\left(5^{\mathrm{R} 2}, 10^{\mathrm{R} 3}, 4^{\mathrm{R} 4}\right)$ have the long acyl chain-C12 in 6180 and C10 in S. pennellii LA0716-at the $\mathrm{R}_{3}$ position, with $\mathrm{C} 4$ or $\mathrm{C} 5$ chains at $\mathrm{R}_{2}$ and $\mathrm{R}_{4}$ (Supplementary Fig. 1b). Furthermore, each detected BIL6180 acylsucrose peak was composed of multiple isomers differing by branched acyl chain types. Liquid chromatography-based separation resulted in the co-elution of a mixture of S3:15 $(5,5,5)$ structural isomers (Supplementary Fig. 1b), and their NMR spectra revealed either iC5 or aiC5 at each of the three acylation positions. In addition, two co-eluting $\mathrm{S} 3: 22(5,5,12)$ isomers (Supplementary Fig. 1b) differed by the presence of iC5 or aiC5 acyl chains at the $R_{4}$ position. Taken together, these results indicate that BIL6180 accumulates pyranose ring acylated triacylsucroses lacking furanose ring acylation, which are similar to those found in the S. pennellii LA0716 parent (Fig. 1b). The similarity between the acylsucroses from BIL6180 and S. pennellii LA0716 led us to hypothesize that BIL6180 could be utilized to characterize the $S$. pennellii acylsucrose biosynthetic pathway.

Sp-ASAT2 and Sp-ASAT3 make P-type acylsucroses. BIL6180 contains three $S$. pennellii introgressions, including regions on chromosome 4 and 11 that contain Sp-ASAT2 and Sp-ASAT3, respectively (Fig. 1c). These are orthologs of the $S$. lycopersicum ASAT genes Sl-ASAT2 and Sl-ASAT3, which encode enzymes catalyzing the second and third steps of the cultivated tomato acylsucrose biosynthetic pathway ${ }^{22,27}$. This led to the hypothesis that Sp-ASAT2 and Sp-ASAT3 contribute to biosynthesis of Ptype acylsucroses in S. pennellii LA0716. To determine whether the $S$. pennellii loci that led to P-type acylsucrose accumulation in BIL6180 are dominant or recessive, we crossed homozygous BIL6180 with the parent S. lycopersicum M82 and analyzed the leaf acylsugar profiles of the F1 progeny by LC-ToF MS (Supplementary Fig. 2). Both F- and P-type acylsucroses accumulated in the F1 plants, indicating that the loci contributing to F- and Ptype acylsucroses are co-dominant, which led us to design transgenic experiments to test our hypothesis.

We engineered $S$. lycopersicum M82 plants by simultaneously expressing the $S p$-ASAT2 and $S p$-ASAT3 coding regions driven by the type I/IV trichome specific promoters ${ }^{22,29}$ of Sl-ASAT2 and Sl-ASAT3, respectively (Fig. 2a). As predicted, the P-type acylsucroses S3:15 (5, 5, 5)-P and S3:22 (5, 5, 12)-P were produced in the regenerated transgenic T0 plants, but not in the parental M82 (Fig. 2b and Supplementary Fig. 3). Similar to the F1 double heterozygotes (Supplementary Fig. 2), the F-type acylsucroses were also detected (Fig. $2 \mathrm{~b}$ and Supplementary Fig. 3). This result provides strong evidence that $S p-A S A T 2$ and $S p$-ASAT3 are sufficient for P-type acylsucrose production in $S$. pennellii and BIL 6180, and led us to characterize their enzymatic activities.

Reversed reaction order leads to P-type acylsucroses. To test their role in P-type acylsucrose biosynthesis, we performed in vitro assays using purified Sp-ASAT2 and Sp-ASAT3 proteins. Published work demonstrated that Sl-ASAT2 acylates the monoacylsucrose $\mathrm{S} 1: 5$ (iC5 $^{\mathrm{R} 4}$ ) pyranose ring $\mathrm{R} 3$ position to produce a diacylsucrose ${ }^{22}$. In contrast, Sp-ASAT2 does not use the monoacylated sucrose as a substrate with any of the acyl-CoA donors $^{22}$. Surprisingly, the S. pennellii LA0716 ortholog of the third enzyme in the cultivated tomato pathway-Sp-ASAT3utilized monoacylated sucrose and $\mathrm{iC} 5-\mathrm{CoA}$ donor substrates to produce a diacylated product with the second chain on the pyranose ring 27 . NMR analysis of the purified diacylsucrose S2:10 $\left(\mathrm{iC} 5, \mathrm{iC} 5^{\mathrm{R} 4}\right)$ produced by the combination of of Sl-ASAT1 and Sp-ASAT3 revealed that the second chain is acylated at the $R_{2}$ position of the pyranose ring (Supplementary Fig. 4). Finally, HPLC analysis revealed that the diacylsucrose $\mathrm{S} 2: 10\left(\mathrm{iC}^{\mathrm{R} 2}\right.$, $\mathrm{iC}^{\mathrm{R} 4}$ ) made by Sp-ASAT3 did not co-elute with the Sl-ASAT2 product $\mathrm{S} 2: 10$ (aiC5 ${ }^{\mathrm{R} 3}, \mathrm{iC} 5^{\mathrm{R} 4}$ ) (Fig. 3a), supporting the hypothesis that the two diacylsucroses made by Sp-ASAT3 and Sl-ASAT2 are different compounds.

The production of S2:10 (iC5 $\left.5^{\mathrm{R} 2}, \mathrm{iC}^{\mathrm{R} 4}\right)$ by Sp-ASAT3 led us to hypothesize that the $S$. pennellii LA0716 ASAT2 ortholog uses this diacylsucrose as an acceptor substrate to produce the P-type 
a

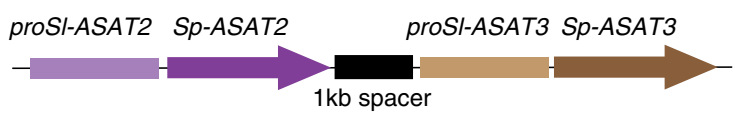

b

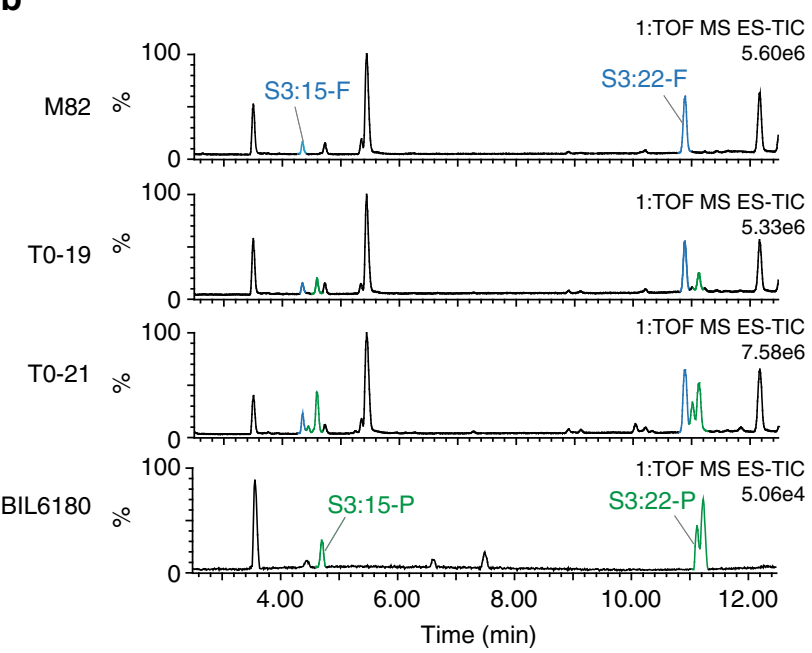

Fig. 2 Transgenic expression of Sp-ASAT2 and Sp-ASAT3 causes trichomes of cultivated tomato $S$. lycopersicum M82 to produce P-type acylsucroses. a Schematic representation of the transgenic locus used to express Sp-ASAT2 and Sp-ASAT3, driven by the SI-ASAT2 and SI-ASAT3 promoters, respectively. A $1 \mathrm{~kb}$ spacer separates these genes. $\mathbf{b}$ TO first generation transgenic plants accumulate the F-type acylsucroses S3:15-F and S3:22-F, which are produced in M82, as well as the P-type acylsucroses S3:15-P and S3:22-P found in BIL6180. The $Y$-axes represent abundance of each peak relative to the most abundant ion intensity in each LC-MS run

acylsucroses found in this species and in BIL6180 (Fig. 1b). Indeed, Sp-ASAT2 produced triacylsucroses (Fig. 3b) with the same positive-ion mode MS fragment ion masses (Supplementary Fig. 1a) and chromatographic retention times (Fig. 3b) as the BIL6180 acylsucroses. These results are consistent with the hypothesis that the in vitro products and in vivo metabolites have the same structures. Based on these results we deduce that $\mathrm{Sp}$ ASAT2 adds the third acyl chain to the pyranose ring $\mathrm{R}_{3}$ position, producing the triacylsucroses $\mathrm{S} 3: 15\left(5^{\mathrm{R} 2}, 5^{\mathrm{R} 3}, 5^{\mathrm{R} 4}\right)$ and $\mathrm{S} 3: 22\left(5^{\mathrm{R} 2}\right.$, $\left.12^{\mathrm{R} 3}, 5^{\mathrm{R} 4}\right)$. The diacylsucrose $\mathrm{S} 2: 10\left(\mathrm{iC} 5^{\mathrm{R} 2}, \mathrm{iC}^{\mathrm{R} 4}\right)$ is not an acyl acceptor substrate for any characterized cultivated tomato enzyme (Sl-ASAT1, Sl-ASAT2, Sl-ASAT3 or Sl-ASAT4). The combined biochemical evidence argues that S. pennellii LA0716 produces triacylsucroses with decorations entirely on the pyranose ring by the stepwise reactions of Sp-ASAT1, SpASAT3 and Sp-ASAT2. Therefore, the S. pennellii LA0716 acylsucrose biosynthetic pathway can be drawn side by side with that of cultivated tomato $S$. lycopersicum by reversing the enzyme action order of ASAT2 and ASAT3 (Fig. 3c).

We tested this 'flipped pathway' hypothesis by transgenic RNA interference (RNAi) silencing of Sp-ASAT3 in BIL6180. We expected greatly reduced acylsugar accumulation in these silenced lines if Sp-ASAT3 catalyzes the second step in the S. pennellii LA0716 pathway. This hypothesis is based on past work that showed that monoacylated sucrose $\mathrm{S} 1: 5 \quad\left(\mathrm{iC} 5^{\mathrm{R} 4}\right)$ does not accumulate to detectable levels in Sl-ASAT2 silenced S. lycopersicum M82 22 . Indeed, most of the BIL6180 T0 transgenic lines had reduced total acylsugar levels (Supplementary Fig. 5), with diacylsucroses undetected in the silenced lines. These transgenic plant results reinforce the in vitro enzyme assays showing that $\mathrm{Sp}$ ASAT3 catalyzes the second biosynthetic step of acylsucroses in BIL6180 and S. pennellii LA0716 (Fig. 3c).
Amino acids that contribute to F- and P-type ASATs divergence. We used a phylogeny-guided approach ${ }^{22,25}$ to identify amino acid substitutions responsible for the differences in activities of the orthologous ASAT2 and ASAT3 enzymes in cultivated tomato and S. pennellii LA0716. This approach benefited from the observation that both $S p$-ASAT2 and Sp-ASAT3 share more than $92 \%$ identity at the amino acid level with their orthologs in cultivated tomato. In addition, ASAT2 orthologs exist in wild tomato species that fall within the Solanum tomato clade that includes cultivated tomato and $S$. pennellii ${ }^{37}$. We tested the activities of ASAT2 variants from these wild tomato species using $\mathrm{S} 1: 5\left(\mathrm{iC}^{\mathrm{R} 4}\right)$ and $\mathrm{S} 2: 10\left(\mathrm{iC} 5^{\mathrm{R} 2}, \mathrm{iC} 5^{\mathrm{R} 4}\right)$ as acyl acceptor substrates. As shown in Fig. $4 \mathrm{a}$, the presence of Gly at position 304 correlates with the ability of ASAT2 to use $\mathrm{S} 2: 10\left(\mathrm{iC}^{\mathrm{R} 2}, \mathrm{iC} 5^{\mathrm{R} 4}\right)$ as a substrate. In contrast, Gln ${ }^{135}$ and $\mathrm{Tyr}^{136}$ are found in ASAT2 enzymes that use $\mathrm{S} 1: 5\left(\mathrm{iC} 5^{\mathrm{R} 4}\right.$ ) as a substrate (Fig. 4a). In vitro mutagenesis of $\mathrm{C} 304 \mathrm{G}$ in Sl-ASAT2 led to the ability to convert $\mathrm{S} 2: 10\left(\mathrm{iC} 5^{\mathrm{R} 2}\right.$, $\left.\mathrm{iC}^{\mathrm{R} 4}\right)$ to the P-type triacylsucrose $\mathrm{S} 3: 22\left(\mathrm{iC} 5^{\mathrm{R} 2}, \mathrm{nC} 12^{\mathrm{R} 3}, \mathrm{iC} 5^{\mathrm{R} 4}\right)$ (Fig. 4b). This single amino acid substitution made the enzyme more promiscuous because it retained the Sl-ASAT2 ability to use $\mathrm{S} 1: 5\left(\mathrm{iC} 5^{\mathrm{R} 4}\right)$ as a substrate (Supplementary Fig. 6). The predicted change in substrate specificity was seen following in vitro mutagenesis of Sp-ASAT2 to generate the mutant with both substitutions H135Q and C136Y: this mutant enzyme gained the ability to utilize $\mathrm{S} 1: 5\left(\mathrm{iC}^{\mathrm{R} 4}\right)$ as the acceptor, producing the diacylsucrose $\mathrm{S} 2: 10\left(\mathrm{aiC} 5^{\mathrm{R} 3}, \mathrm{iC}^{\mathrm{R} 4}\right)$, as did wild-type Sl-ASAT2 (Fig. 4c). Mutation of both amino acids (135 and 136) was required for complete conversion: both single mutants of $\mathrm{Sp}$ ASAT2 H135Q and C136Y showed a residual ability to use S1:5 $\left(\mathrm{iC}^{\mathrm{R} 4}\right)$ as a substrate (Supplementary Fig. 7).

To assess whether the residues associated with ASAT2 F- or Ptype activity have a similar function in other tomato relatives, the F-type ASAT2 from S. arcanum LA2172 and the P-type ASAT2 from S. pennellii LA1926 were cloned and subjected to in vitro mutagenesis. Indeed, S. arcanum LA2172-ASAT2 with substitution C304G gained the P-type ability to acylate $\mathrm{S} 2: 10\left(\mathrm{iC} 5^{\mathrm{R} 2}\right.$, $\mathrm{iC}^{\mathrm{R} 4}$ ) (Supplementary Fig. 8a). Similar to the S. pennellii LA0716 Sp-ASAT2 mutant with both residue changes H135Q and C136Y, substitution of P135Q and C136Y in S. pennellii LA1926-ASAT2 enabled the enzyme to catalyze the F-type activity by acylating $\mathrm{S} 1: 5\left(\mathrm{iC} 5^{\mathrm{R} 4}\right.$ ) (Supplementary Fig. 8b). These results corroborated those obtained by mutagenesis of Sl-ASAT2 and Sp-ASAT2.

We employed a similar approach to identify amino acid residues that shape ASAT3 enzyme activities. A previous study documented 35 ASAT3 variants from $S$. habrochaites that belong to two clades-F- and P-type-which catalyze furanose and pyranose ring acylation similar to Sl-ASAT3 and Sp-ASAT3, respectively ${ }^{27}$. A survey of amino acids from six Sh-ASAT3 from each enzyme group (Fig. 5a), revealed a correlation between residues at positions 161, 162, and 289 and enzyme types (Fig. 5a). We tested the importance of these amino acids by making the Sl-ASAT3 triple mutant with $\mathrm{Y} 161 \mathrm{H}, \mathrm{C} 162 \mathrm{~S}$ and T289V: this engineered protein gained the ability to acylate S1:5 $\left(\mathrm{iC} 5^{\mathrm{R} 4}\right)$, as seen in P-type ASAT3 enzymes (Fig. 5b). This mutant was promiscuous for acceptor substrate, maintaining the ability to acylate the furanose ring of $\mathrm{S} 2: 10\left(\mathrm{aiC} 5^{\mathrm{R} 3}, \mathrm{iC} 5^{\mathrm{R} 4}\right)$ (Supplementary Fig. 6b). Interestingly, each of the three Sl-ASAT3 single-residue mutants gained a partial ability to acylate $\mathrm{S} 1: 5\left(\mathrm{iC} 5^{\mathrm{R} 4}\right)$, though less well than the triple mutant (Supplementary Fig. 9a). The SpASAT3 triple mutant-with the reciprocal changes H161Y, S162C, and V289T-lost the ability to acylate $\mathrm{S} 1: 5\left(\mathrm{iC}^{\mathrm{R} 4}\right)$ without gaining F-type ASAT3 activity.

Further sequence comparison of the twelve ShASAT3 variants revealed that the residues at position 354, 381, and 382 correlate with enzyme group differences (Fig. 5a). In Sl-ASAT3, these residues are $\mathrm{Leu}^{354}, \mathrm{His}^{381}$, and Pro ${ }^{382}$, whereas in Sp-ASAT3 

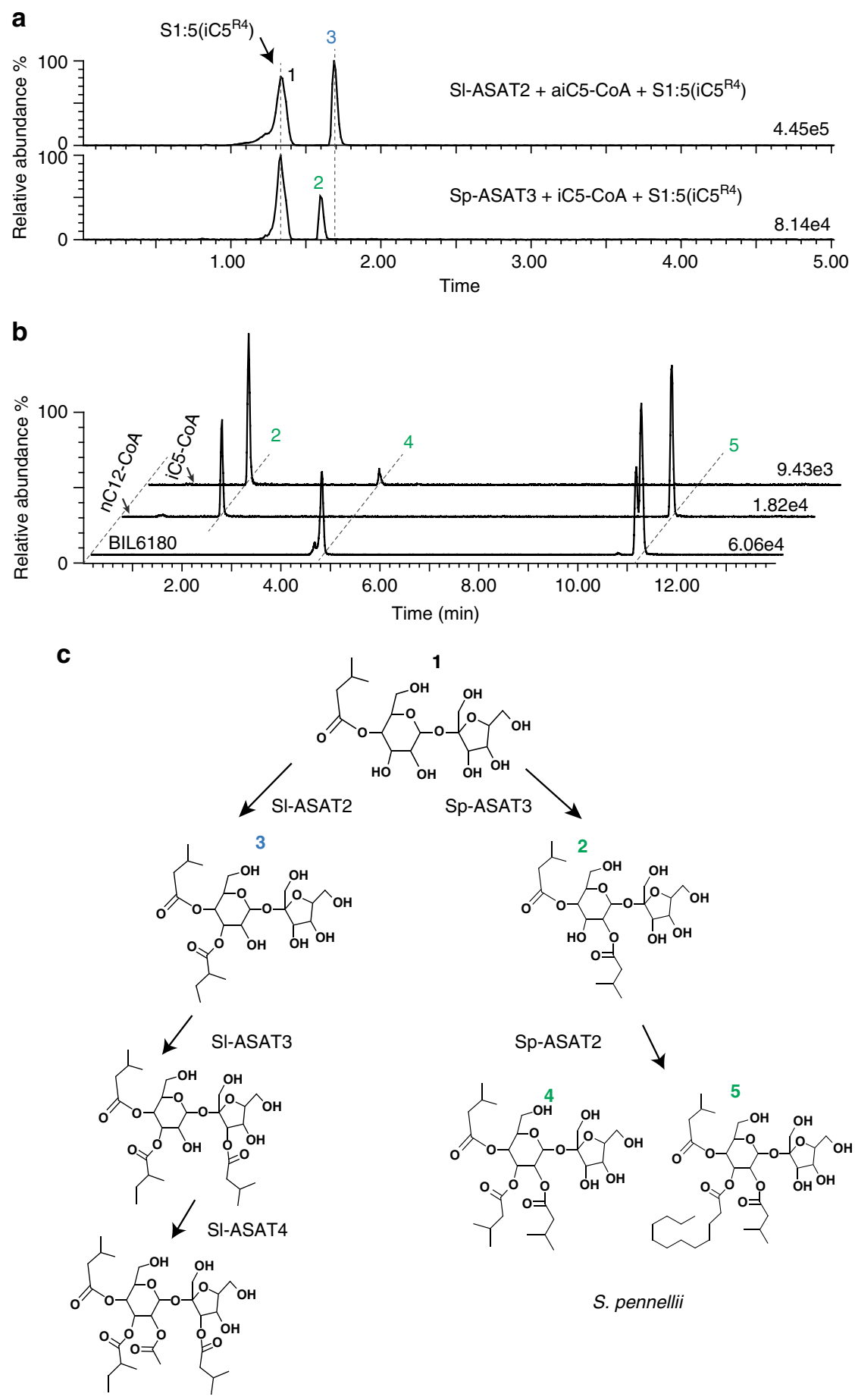

S. lycopersicum

Fig. 3 Sp-ASAT2 and Sp-ASAT3 produce P-type acylsucroses via a "flipped pathway". a S. pennellii LA0716 wild tomato Sp-ASAT3 acylates S1:5 (iC5R4) and produces a diacylsucrose product (peak 2) with a retention time distinct from that made by cultivated tomato SI-ASAT2 (peak 3). b Sp-ASAT2 converts the S2:10 ( $i C 5^{R 2}$, iC5 ${ }^{R 4}$ ) diacylsucrose produced by Sp-ASAT3 (peak 2) to triacylsucroses with the same retention times as those produced by BIL6180 (peaks 4 and 5). "iC5-CoA" and "nC12-CoA" refer to the two different acyl donor substrates used in the two enzyme reactions. c The "flipped" acylsucrose biosynthetic pathways in S. lycopersicum and S. pennellii LA0716. The numbers on top of each compound refer to the chromatographic peaks in $\mathbf{a}$ and $\mathbf{b}$

they are $\mathrm{Val}^{353}$ and $\mathrm{Ser}^{380}$, with one amino acid deletion adjacent to position 380 compared with Sl-ASAT3. To test whether these residues are associated with the ability of ASAT3 to acylate the furanose ring of S2:10 (aiC5 $\left.{ }^{\mathrm{R} 3}, \mathrm{iC} 5^{\mathrm{R} 4}\right)$, Sp-ASAT3 was mutagenized to generate the following changes: V353L and S380HP. Indeed, the mutagenized enzyme gained the F-type ability to acylate $\mathrm{S} 2: 10\left(\mathrm{aiC} 5^{\mathrm{R} 3}, \mathrm{iC} 5^{\mathrm{R} 4}\right)$ in vitro (Fig. 5c). Meanwhile, we detected a small amount of F-type activity for Sp-ASAT3 mutants with the single substitutions V353L or S380HP (Supplementary Fig. 9b). While these amino acid substitutions correlate withand predict-enzymatic activity in the twelve ASAT3 proteins documented in Fig. 5, there are 10 P-type ASAT3 enzymes that 

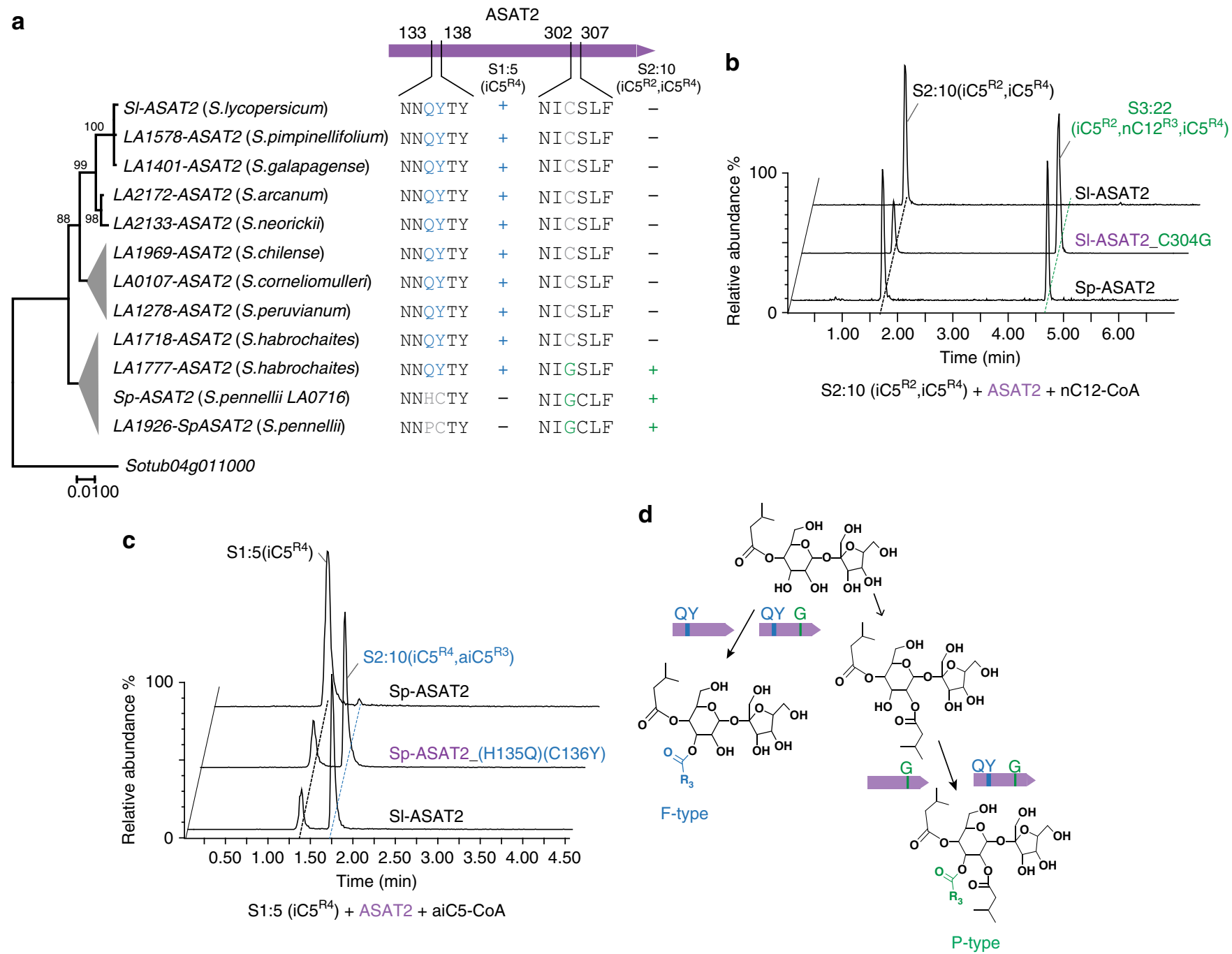

Fig. 4 Identification of key residues that contribute to switching ASAT2 activities between productions of F- or P-type acylsucroses. a Correlation between ASAT2 enzyme activities and amino acid sequences for various tomato species. The maximum likelihood phylogenetic gene tree was obtained using the nucleotide sequences of ASAT2 variants, with the ASAT2 homolog (Sotub04g011000) in S. tuberosum serving as outgroup. The bootstrap values were obtained with 1000 replicates. The nodes with bootstrap values lower than 70 were collapsed. The ability of different ASAT2 to use acyl acceptor substrates $\mathrm{S} 1: 5\left(\mathrm{iC}^{\mathrm{R} 4}\right)$ or $\mathrm{S} 2: 10\left(\mathrm{iC}^{\mathrm{R} 2}, \mathrm{iC}^{\mathrm{R} 4}\right)$ is shown by " + ", while "-" indicates no detected enzyme activity. Presence of $\mathrm{Q}^{135} \mathrm{Y}^{136}$ and $\mathrm{G}^{304}$ correlate with activity with S1:5 and 2:10 acceptor substrates, respectively. b Results of in vitro assays performed with the site-directed mutant enzymes. SI-ASAT2 with the residue substitution C304G produced the P-type triacylsucrose product that has the same chromatographic retention time with Sp-ASAT2's product using S2:10 $\left(\mathrm{iC} 5^{\mathrm{R} 2}, \mathrm{iC} 5^{\mathrm{R} 4}\right)$ as the substrate. c Sp-ASAT2 with the dual residue substitutions $\mathrm{H} 135 \mathrm{Q}$ and $\mathrm{C} 136 \mathrm{Y}$ produced the diacylsucrose product that has the same chromatographic retention time with SI-ASAT2's product using S1:5 (iC5 ${ }^{\mathrm{R} 4}$ ) as the substrate. $\mathbf{d}$ Interpretation of the results of parts $\mathbf{b}$ and $\mathbf{c}$, showing the key amino acids associated with F- and P-type ASAT2 enzyme activities

do not follow the $\mathrm{Val}^{353}$ and $\mathrm{Ser}^{380}$ pattern (gray circles in Supplementary Fig. 12).

Taken together, this combined comparative biochemical and mutagenesis analysis revealed multiple ASAT2 and ASAT3 residues that contribute to the divergence of ASAT2 and ASAT3 enzyme activities, which in turn result in F- or P-type acylsucroses. As expected for amino acids that influence substrate specificity, homology modeling with the trichothecene 3-Oacetyltransferase-acyl CoA complex structure (PDB code 3B2S $)^{38}$ predicted that these residues surround the reaction center (Supplementary Fig. 10).

Acylsucrose pathway divergence in the Solanum tomato clade. We previously characterized the distribution pattern of F- or Ptype acylsucroses in the tomato group ${ }^{27}$. The results described above led to the hypothesis that the distribution of F- and P-type acylsucroses across this group reflects the divergence of the acylsucrose pathway in the tomato clade driven by ASAT2 and ASAT3. To test this hypothesis, we sequenced the ASAT2 and ASAT3 genes of species across this group and determined whether the plant acylsucrose phenotypes can be inferred using ASAT2 and ASAT3 genotypes.

In the tomato subclade ranging from $S$. lycopersicum through $S$. corneliomulleri, all characterized ASAT2 proteins in this tomato subclade possessed only F-type enzymatic activities (Fig. 4a). In addition, sequenced ASAT3 orthologs from five species in this subclade all have the F-type activity-associated residues Leu ${ }^{353}$ and $\mathrm{His}^{381} \mathrm{Pro}^{382}$ (see bottom of Supplementary Fig. 12), which predicts that all of these ASAT3 orthologs have F-type enzymatic activities. The in vitro enzyme activities were tested for three ASAT3 from S. arcanum (LA2172-ASAT3_1), S. huaylasense 
a

ASAT3
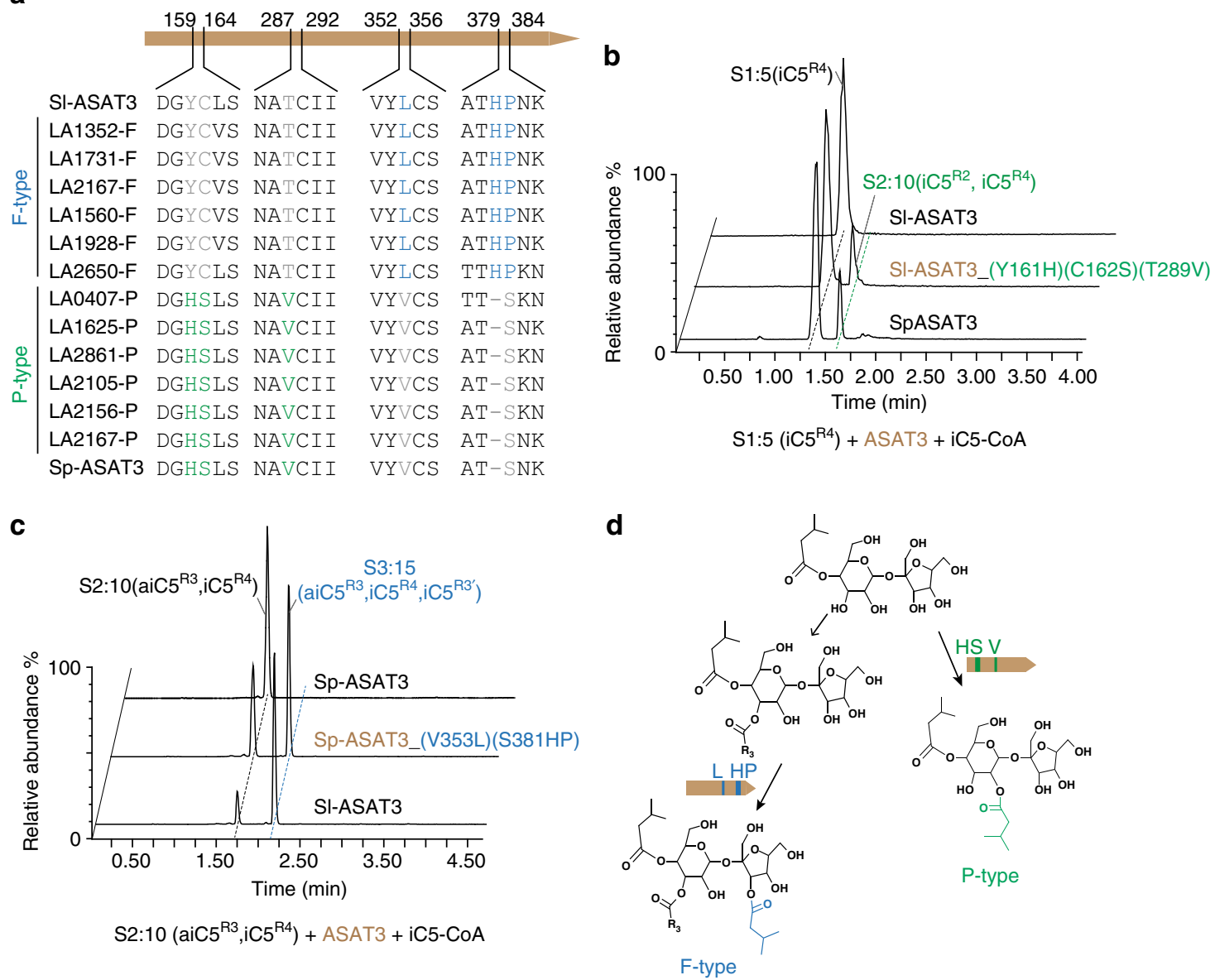

Fig. 5 Identification of residues that confer P-type activity to SI-ASAT3 or F-type activity to Sp-ASAT3. a Amino acid sequences of ASAT3-P or -F alleles from different $S$. habrochaites accessions revealed residues that correlate with each enzyme type, and these are color coded. Blue amino acids are associated with F-type and green residues with P-type activity. $\mathbf{b}$ The results of in vitro assays performed with the two parental enzymes and the triple mutant created by site-directed mutagenesis of SI-ASAT3 enzyme. The SI-ASAT3 protein with three residue substitutions-Y161H, C162S, and T289V-

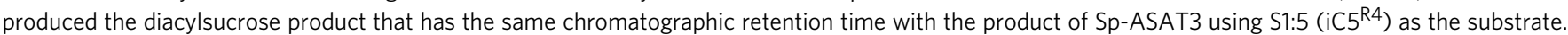
c The double amino acid position mutant Sp-ASAT3-with V353L and S380HP-produced the triacylsucrose product that has the same chromatographic retention time with the product of SI-ASAT3 using S2:10 (aiC $5^{\mathrm{R} 3}$, iC $5^{\mathrm{R} 4}$ ) as the substrate. $\mathbf{d}$ Interpretation of the results of parts $\mathbf{b}$ and $\mathbf{c}$, showing the key amino acids associated with F- and P-type ASAT3 enzyme activities

(LA1364-ASAT3), and $S$. peruvianum (LA1278-ASAT3_1). Indeed, they have the F-type activity, acylating $\mathrm{S} 2: 10$ (aiC5 ${ }^{\mathrm{R} 3}$, $\mathrm{iC}^{\mathrm{R} 4}$ ) (Supplementary Fig. 13, left panel). The combination of ASAT2-F and ASAT3-F alleles predicts that species in this group would only have the F-type acylsucrose biosynthetic pathway (Fig. 6b), matching the previously described F-type trichome acylsucroses of $S$. arcanum, $S$. neorickii, S. huaylasense, S. peruvianum, and S. corneliomulleri ${ }^{27}$. Interestingly, LA1364ASAT3 and LA1278-ASAT3_1 have residues His ${ }^{161}$ and Ser ${ }^{162}$, respectively; each was associated with the P-type activity from the $S$. habrochaites and $S$. pennellii analysis. Consistent with this observation, these two ASAT3 enzymes are promiscuous, showing a minor P-type activity to acylate the $\mathrm{R}_{2}$ position of $\mathrm{S} 1: 5\left(\mathrm{iC} 5^{\mathrm{R} 4}\right.$ ) (Supplementary Fig. 13, right panel).

In contrast to other tomato species, only P-type acylsucroses were detected in $S$. pennellii accessions ${ }^{27}$, suggesting that the 'LA0716 biosynthetic pathway' alone is present in these accessions. Indeed, all ASAT3 isoforms isolated from ten $S$. pennellii accessions only have the residues $\mathrm{His}^{161} \mathrm{Ser}^{162}$ and $\mathrm{Val}^{289}$, which are associated with P-type activity (Fig. 6a and Supplementary Fig. 12). This suggests that the uniform P-type ASAT3 activity would direct the pathway to produce P-type acylsucroses (Fig. 6b).
In addition, ASAT2 cloned from five S. pennellii accessions originating from south Peru (Supplementary Fig. 14) possess the residue $\mathrm{Gly}^{304}$, which is associated with P-type activity (Supplementary Fig. 11). However, S. pennellii ASAT2 cloned from northern Peruvian accessions (Supplementary Fig. 11) have both F- and P-type activity-associated residues (Supplementary Fig. 14), which predicts that they have hybrid F- and P- type enzyme activities: this combination is reminiscent of $S$. habrochaites LA1777-ASAT2 (Fig. 4a). Although this ASAT2 F-type activity would enable the pathway to branch and make both F- and P-type acylsucroses, the F-type diacylsucrose intermediates are not expected to be acylated further due to a lack of ASAT3 F-type activity (Fig. 6b). This presumably is why no F-type acylsucroses were found in these northern Peruvian accessions ${ }^{27}$.

S. habrochaites is the only species in the tomato group with accessions demonstrated to accumulate a mixture of F- and P-type acylsucroses 27 , providing information regarding the evolutionary relationship of the F- and P- type acylsucroses pathways. Our previous study identified 13S. habrochaites accessions with both the $\mathrm{F}$ and $\mathrm{P}$ alleles of $\mathrm{ASAT} 3^{27}$. To extend this study, we further cloned sequences for ASAT2 enzymes from six of the S. habrochaites accessions that harbored both ASAT3-F 
a
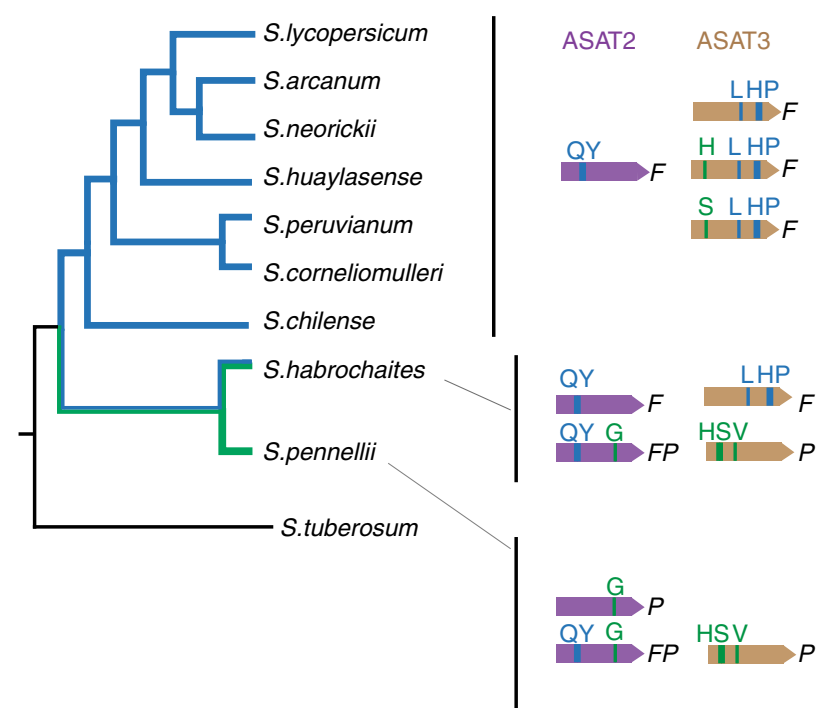

Genotype

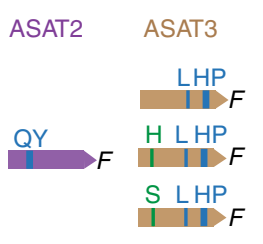

Acylsucrose phenotype

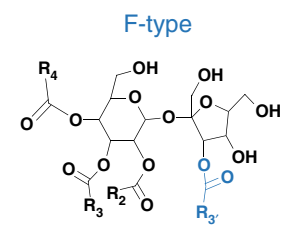

-type

b

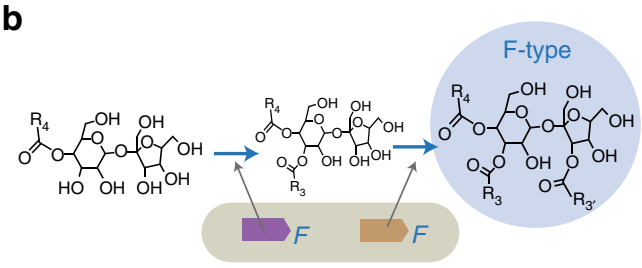

S. Iycopersicum through S. chilense
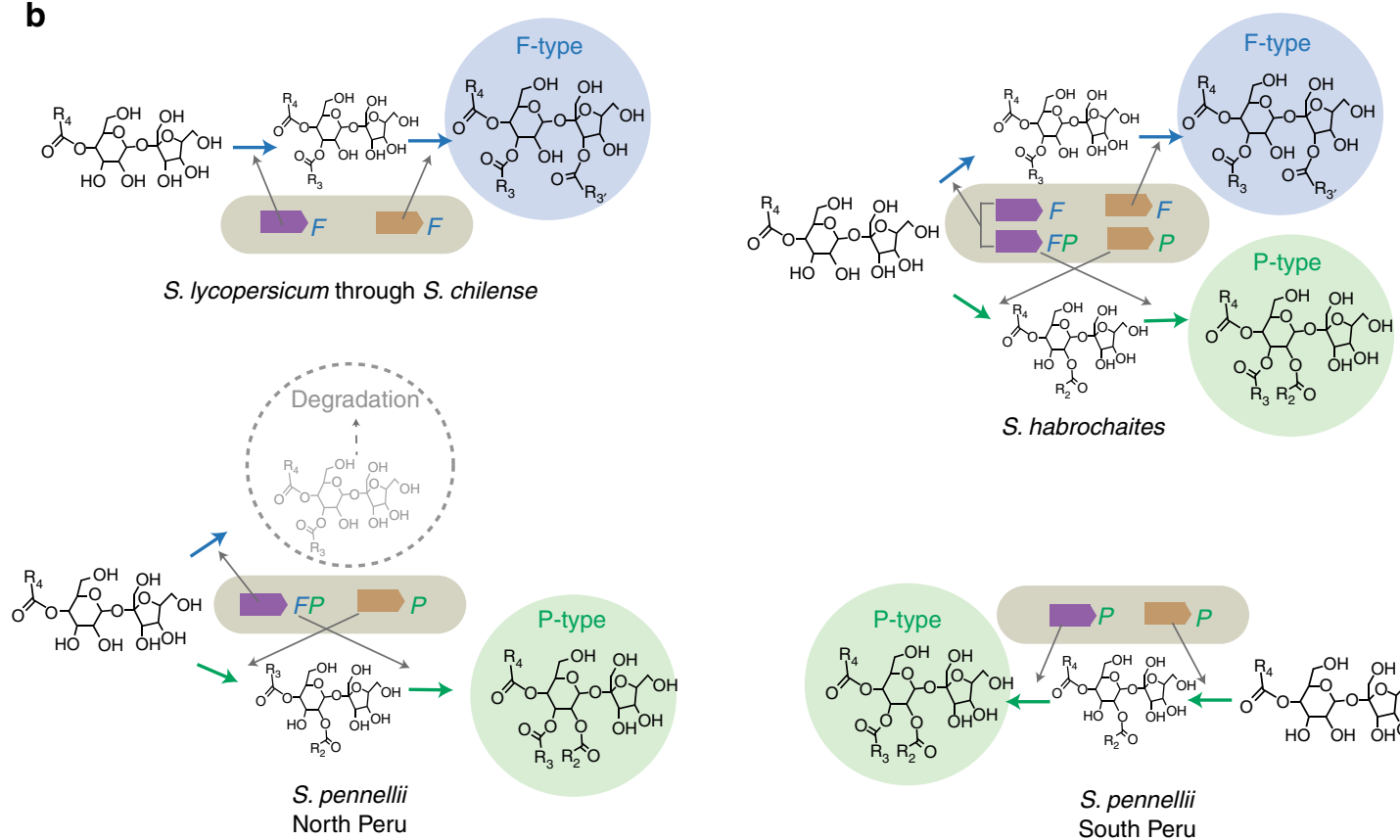

S. habrochaites

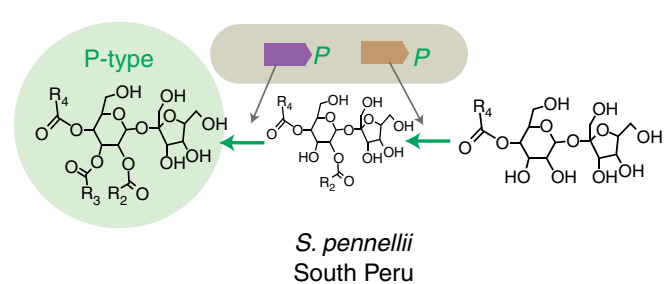

Fig. 6 Solanum tomato group ASAT2 and ASAT3 variant proteins in a phylogenetic context. a ASAT2 and ASAT3 variants cloned from different wild tomato species are assigned as $\mathrm{F}$ - or P-protein types based on residues identified by in vitro mutagenesis. Amino acids associated with $\mathrm{F}$ - or $\mathrm{P}$-type activities are in blue and green, respectively. See Supplementary Figs. 11 and 12 for detailed gene phylogenies. The plant trichome F- or P- acylsucrose phenotypes are based on published results. There are NMR structural data for acylsugars from S. lycopersicum, S. habrochaites and S. pennellii ${ }^{28,30}$, while LC/MS data exist for all species listed ${ }^{27}$. b Summary of the types of ASAT and product combinations found in the tomato clade. The combination of ASAT2 and ASAT3 enzyme types in different tomato species was inferred based on the activity-associated residues as shown in a. Degradation of the dead end diacylsucrose is based on published results for loss of function S. lycopersicum ASAT3 ${ }^{27}$ and characterization of acylsucrose hydrolases in S. lycopersicum and S. pennelliii ${ }^{30}$

and ASAT3-P, including accessions LA1772, LA1978, LA2156, LA2650, LA2722, and LA2861. We identified at least two ASAT2 alleles from each of the selected $S$. habrochaites accessions; in each case, one ASAT2 harbors both F- and P-type residues and the other one only possesses the F-type residues (Supplementary Fig. 11). The combination of ASAT2 and ASAT3 with both Fand P-types suggests the co-existence of $\mathrm{F}$ - and P-type acylsucrose biosynthetic pathway in these plants (Fig. 6b), which is consistent with $S$. habrochaites species having the most varied acylsucrose phenotypes across the tomato group described to date 27,29 .

\section{Discussion}

Metabolic innovation events can endow an organism with a greater ability to survive in a challenging environment ${ }^{39,40}$. The mixture of specialized metabolites produced in a plant species or group results from assembly of enzymes into metabolic pathways or networks, and evolution of novel products occurs one step at a time. In this process, change in the activity of a single enzyme to produce a new product can lead to a variety of consequences. The simplest productive outcome is that the next enzyme in the pathway is promiscuous and uses the new product as a substrate, converting it to a product that is slightly different from the 


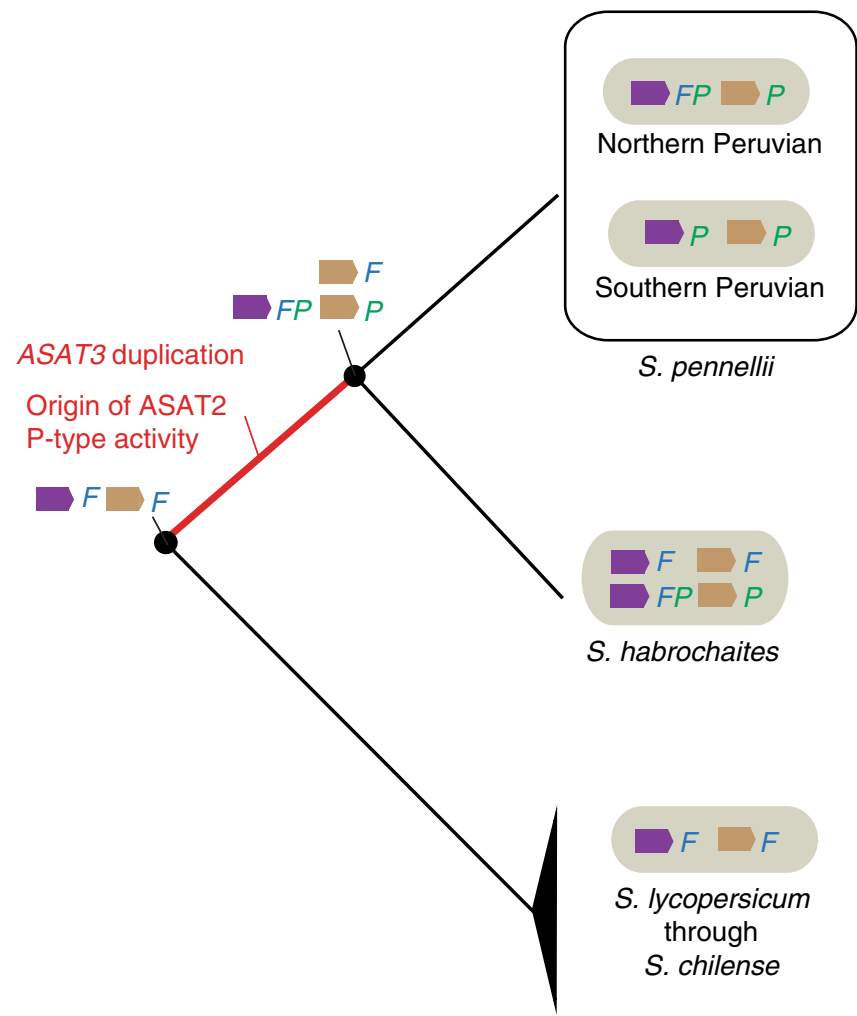

Fig. 7 Summary of results suggesting that P-type ASAT2 and ASAT3 enzyme evolution occurred after the last common ancestor of the tomato clade of Solanum. Ancestral ASAT2 and ASAT3 genotypes were inferred based on the ASAT2 (Supplementary Fig. 11) and ASAT3 (Supplementary Fig. 12) gene phylogenies. It suggests that the ASAT3 duplication event and the origin of ASAT2 P-type activity occurred before the speciation of S. habrochaites and S. pennellii (the red-colored branch) and away from the lineage containing the cultivated tomato S. lycopersicum and its closer relatives

predecessor. A second possibility is that the new product may not be a substrate for any available biosynthetic enzymes, precluding further conversion steps. Alternatively, the new product might be a substrate for an enzyme not normally involved in the predecessor pathway-so called 'silent' or 'occult' metabolism ${ }^{41}$ giving rise to a novel metabolic product. The example described in this paper represents a more complicated case, involving changes in substrate specificity for two enzymes that function in consecutive reactions. ASAT2 and ASAT3, which are the second and third enzymes of the cultivated tomato F-type acylsucrose biosynthetic pathway, evolved new substrate specificities in the $S$. habrochaites/S. pennellii tomato subgroup. This allows the new Sp-ASAT3-P product to become a substrate for Sp-ASAT2-P, leading to reversal of their enzymatic order and pyranose ring $\mathrm{P}$ type triacylsucrose production (Fig. 3c).

Discovery of the enzymes of P-type acylsucrose biosynthesis was facilitated by LC/ToF-MS screening of BILs, each of which contains multiple regions of the $S$. pennellii LA0716 genome in an $S$. lycopersicum genetic background ${ }^{35}$. The P-type acylsucrose producer (BIL6180) has three S. pennellii chromosomal regions, including those containing $S p-A S A T 2$ and $S p$-ASAT3 (Fig. 1c). Evidence that these acylsucrose acyltransferases are sufficient to account for the P-type acylsucrose phenotype came from $S$. lycopersicum M82 transgenic lines expressing both $S p$-ASAT2 and $S p$-ASAT3 in type I/IV trichomes. These accumulated both F- and P-type acylsucroses (Fig. 2), as predicted based on the phenotype of a BIL6180 × wild-type M82 heterozygote (Supplementary
Fig. 2). Results of in vitro enzyme analysis confirmed that these enzymes produce P-type products (Fig. 3). Sp-ASAT3 uses monoacylsucrose $\mathrm{S} 1: 5\left(\mathrm{iC}^{\mathrm{R} 4}\right)$ as an acyl acceptor substrate-as previously reported for Sl-ASAT2-to produce a diacylsucrose product $\mathrm{S} 2: 10\left(\mathrm{iC} 5^{\mathrm{R} 2}, \mathrm{iC} 5^{\mathrm{R} 4}\right)$ that is distinct from any in the previously identified F-type acylsucrose pathway (Fig. 3c). SpASAT2 next converts this product into P-type triacylsucroses. The combination of in vivo and in vitro results demonstrate that the 'flipped pathway' is at the center of divergence of the F- and P-type acylsucrose pathways (Fig. 3c).

Comparative biochemical analysis revealed a relatively small number of ASAT2 and ASAT3 amino acid differences that mediate the change in pathway order. ASAT2 neofunctionalization from F- to P-type enzymatic activity is associated with three amino acid substitutions (Fig. 4). S. pennellii and S. habrochaites accessions contain the ASAT2 change C304G, which is sufficient to promote ASAT2 P-type enzyme activity. In contrast, the phylogenetically more restricted $\mathrm{Gln}^{135} \mathrm{Tyr}^{136}$ to $\mathrm{His}^{135} \mathrm{Cys}{ }^{136}$ change (Supplementary Figs. 11 and 14) abolishes the ASAT2 Ftype activity in $S$. pennellii accessions from southern Peru (including LA0716, the S. pennellii parent of the IL and BIL). The differences between extant P- and F-type ASAT3 isoforms are mediated by changes in multiple amino acids, a theme common in enzyme evolution ${ }^{42}$. At least three residue substitutionsY161H, C162S and T289V-are needed for the modern F-type SlASAT3 to acquire P-type enzyme activity (Fig. 5b). Interestingly, comparison of all amino acids involved in flipping the pathway modeled the Sl-ASAT2 C304 and Sl-ASAT3 T289 to be at the same position in the predicted 3-D structures, near the acyl-CoA site (Supplementary Fig. 10d). These results suggest this position in the binding site as a candidate for future efforts to engineer BAHD acyltransferases for synthetic biology, and for understanding the evolution of form and function of related enzymes.

These results add to past work documenting how inter- and intra-specific variation in ASAT acyl donor promiscuity influence diversification of Solanum trichome acylsucrose products. For example, F-type ASAT2 enzymes from different tomato species specifically vary in preference for iC5-CoA as substrate ${ }^{22}$, without affecting aiC5-CoA or nC12-CoA, thus influencing acyl chain types at the sucrose $\mathrm{R}_{3}$ position ${ }^{28}$. In addition, all $S$. habrochaites F-type ASAT3 isoforms tested utilized iC5-CoA, but only a subset also accepted the long chain nC12-CoA donor ${ }^{27}$. Taken together with the results described above, we conclude that ASAT2 and ASAT3 acyl donor and acceptor promiscuity played roles in the evolution of these BAHD acyltransferases and diversification of trichome metabolite accumulation.

Despite the involvement of two enzymes, the divergence of Fand P-type acylsucrose appears to have occurred relatively recently-over the past 2.5 million years-which was a period of rapid diversification within the Solanum tomato clade ${ }^{43,44}$. We hypothesize that this innovation occurred following divergence leading to the $S$. habrochaites/S. pennellii group and away from the lineage containing the cultivated tomato S. lycopersicum and its closer relatives (shown by a red line in Fig. 7). We base this on two lines of evidence. First, ASAT2 and ASAT3 from the group of closer relatives ( $S$. lycopersicum through S. chilense) exhibit F-type activities, whereas a mix of F- and P-type activities is observed for ASAT2 (Fig. 4) and ASAT3 ${ }^{27}$ from S. habrochaites. This suggests that the F-acylsucrose biosynthetic network is ancestral in the Solanum tomato clade. Second, based on extant ASAT2 (Supplementary Fig. 11) and ASAT3 (Supplementary Fig. 12) coding sequence phylogenetic relationships, only residues associated with F-type activities appear to have existed in the ancestor of wild tomatoes. In contrast, the gene phylogenies suggest that $\mathrm{P}$ type residues emerged in an ancestor of $S$. habrochaites and $S$. pennellii. In addition, characterization of additional ASAT3 
sequences from wild tomato species other than $S$. habrochaites reinforce the hypothesis that ASAT3 duplication occurred after the last common ancestor of the extant tomato group and before S. habrochaites and S. pennellii speciation ${ }^{27}$ (red line in Fig. 7).

While we have insufficient resolution to reconstruct the order of events leading to evolution of the P-type acylsucrose pathway, our results led to a general model for P-type pathway evolution, where promiscuity features prominently. For example, the C304G mutation is associated with P-type activity: mutagenesis of SlASAT2 causes acquisition of P-type activity, while retaining the original F-type activity (Fig. 4b and Supplementary Fig. 6). This is not a merely a laboratory anomaly as there are ASAT2 natural variants that have both F- and P-type activities. For example, LA1777-ASAT2 cloned from S. habrochaites acylates both S1:5 $\left(\mathrm{iC}^{\mathrm{R} 4}\right)$ and $\mathrm{S} 2: 10\left(\mathrm{aiC} 5^{\mathrm{R} 3}, \mathrm{iC}^{\mathrm{R} 4}\right)$ (Fig. 4a). Similarly, substitution of Sl-ASAT3 with three amino acids associated with ASAT3-P activity $(\mathrm{Y} 161 \mathrm{H}, \mathrm{C} 162 \mathrm{~S}, \mathrm{~T} 289 \mathrm{~V})$, led to $\mathrm{S} 1: 5\left(\mathrm{iC}^{\mathrm{R} 4}\right)$ acylation on the pyranose ring, while retaining F-type activity (Supplementary Fig. 6). In fact, single substitution of each of these amino acids led to low detectable amounts of Sp-ASAT3 activity (Supplementary Fig. 9a, compare top three chromatograms with the bottom one). The promiscuity of such single or multiple mutants could have potentiated evolution of the ancestral enzyme, leading to production of small amounts of P-type diacylsucroses. In this scenario, accumulation of other mutations to the potentiated enzymes would eventually lead to improved catalytic activity, and a shift to the "purer" P-type activity seen in S. pennellii and S. habrochaites. ASAT2-P refinement by losing F-type activity presumably involved changes at positions 135 and 136 of Sl-ASAT2: $\mathrm{Gln}^{135}$ to (His or Pro) ${ }^{135}$ and $\mathrm{Tyr}^{136}$ to Cys ${ }^{136}$, based on extant sequences shown in Supplementary Fig. 11.

The results of this study leave interesting questions unanswered. For example, when did acylsugar degrading acylhydrolases $^{30}$ evolve and did these enzymes influence emergence or refinement of P-type enzymes? Did emergence of the ASAT3-P activity, which acylates pyranose at the same $R_{2}$ position as does the F-type pathway ASAT4 acetyltransferase, contribute to the ASAT4 gene inactivation events in ancestors of northern Ecuadoran S. habrochaites accessions $\mathrm{s}^{29}$ and S. pennellii LA0716 ${ }^{26}$ ? Do differences in sucrose acylation patterns influence accumulation of acylglucoses in southern vs. northern S. pennellii accessions ${ }^{45}$ ? Thus, this work on variation in two acyltransferases opens up opportunities to study evolution of epistatic interactions between three or more enzymes in tomato and more broadly in the Solanaceae.

\section{Methods \\ Plant growth and trichome acylsugar extraction. Tomato wild species seeds were obtained from the C.M. Rick Tomato Genetic Resource Center (http://tgrc/ ucdvis.edu) and S. pennellii BIL seeds were from Dr. Dani Zamir (Hebrew University of Jerusalem). The tomato seeds were germinated on damp filter paper for one week and then transferred to peat pots. The plants were maintained in a growth chamber for another two to three weeks, with the growth condition as follows: $28^{\circ} \mathrm{C}$ for $16 \mathrm{~h}$ in the light $\left(300 \mu \mathrm{E} \mathrm{m}^{-2} \mathrm{~s}^{-1}\right)$ and $20^{\circ} \mathrm{C}$ for $8 \mathrm{~h}$ in the dark. Trichome metabolites were extracted by submerging the youngest fully developed leaf for $2 \mathrm{~min}$ with gentle agitation in $1 \mathrm{~mL}$ of extraction solution, which contained acetonitrile/isopropanol/water (3:3:2) with $0.1 \%$ formic acid and $10 \mu \mathrm{M}$ propyl-4- hydroxybenzoate as internal standard. Dry leaf weight was measured after the extracted tissue was dried in an oven at $60^{\circ} \mathrm{C}$. We consider each individual plant as a biological replicate. At least three plants from the same line were used for the trichome acylsugar extraction and LC/MS analysis.}

Tomato transformation. To express Sp-ASAT2 and Sp-ASAT3 in S. lycopersicum M82 trichomes, four fragments—a $1.6 \mathrm{~kb}$ region upstream of Sl-ASAT2; the $S p$ ASAT2 gene with a $1 \mathrm{~kb}$ region downstream; $1.7 \mathrm{~kb}$ region upstream of Sl-ASAT3, and the Sp-ASAT3 gene- were amplified and cloned into the vector pENTR/DTOPO (Invitrogen) using the Golden Gate Assembly method ${ }^{46}$. The gene cassette in pENTR/D-TOPO was recombined into the GATEWAY vector $\mathrm{pK} \mathrm{WG}^{47}$ and used for transformation of M82 plants. To suppress Sp-ASAT3 expression in
BIL6180 using RNAi, a fragment of Sp-ASAT3 was amplified from S. pennellii LA0716 cDNA and cloned into pENTR/D-TOPO followed by recombination into the GATEWAY binary vector pHELLSGATE12 ${ }^{48}$ and the resultant plasmid was used to transform BIL6180 plants. Tomato transformation was performed using $A$. tumefaciens strain $\mathrm{AGL} 0^{49}$.

Trichome metabolite extractions were performed with the independent T0 primary transformants and $\mathrm{T} 1$ plants resulted from self-crossing of T0 lines. The primers used for genotyping of the T1 lines are described in Supplementary Table 1.

Analysis of Sp-ASAT3 and Sp-ASAT2 gene expressions. To analyze the mRNAs from Sp-ASAT2 or Sp-ASAT3 in T1 RNAi suppression lines targeting Sp-ASAT3 in BIL6180, the leaves with the same developmental stages as those used for acylsugar extractions were collected. RNA extraction was performed using the Plant RNeasy kit (QIAGEN), and treated with DNase I. The first-strand cDNA synthesis by Superscript II (Invitrogen) used total RNA as templates. Five plants from each independent T1 lines were used as biological replicates. The Quantitative real-time PCR was performed to analyze the gene expression using the elongation factor gene (Solyc06g005060) as an internal control. Primers are listed in Supplementary Table 1. QuantStudio 7 Flex Real-Time PCR System with Fast SYBR Green Master Mix (Applied Biosystems) was used for the analysis. The relative quantification method $\left(2^{-\Delta \Delta \mathrm{Ct}}\right)$ was employed to evaluate the relative transcripts levels.

LC/MS analysis of acylsugars. Both trichome acylsugar extracts and enzyme assay samples were analyzed using a Waters Acquity UPLC system coupled to a Waters Xevo G2-S QToF LC-MS. For all sample injections, ten microliters were used for reverse-phase separation in a fused core Ascentis Express C18 column $(2.1 \mathrm{~mm} \times 10 \mathrm{~cm}, 2.7 \mu \mathrm{m}$ particle size; Sigma-Aldrich) with the column temperature of $40^{\circ} \mathrm{C}$. The LC starting conditions were $95 \%$ solvent A $(0.15 \%$ formic acid in water) and $5 \%$ solvent B (acetonitrile) with a flow rate of $0.3 \mathrm{~mL} / \mathrm{min}$. The $7 \mathrm{~min}$ and 14 min elution gradients - used for separating plant trichome acylsugars and enzyme assay samples-were as following steps. For the $7 \mathrm{~min}$ elution gradient: ramp to $40 \% \mathrm{~B}$ at $1 \mathrm{~min}$, then to $100 \% \mathrm{~B}$ at $5 \mathrm{~min}$, hold at $100 \% \mathrm{~B}$ to $6 \mathrm{~min}$, return to $95 \% \mathrm{~A}$ at $6.01 \mathrm{~min}$ and hold until $7 \mathrm{~min}$. For the $14 \mathrm{~min}$ elution gradient: ramp to $35 \% \mathrm{~B}$ at $1 \mathrm{~min}$, then to $85 \% \mathrm{~B}$ at $12 \mathrm{~min}$, ramp to $100 \% \mathrm{~B}$ at $12.01 \mathrm{~min}$, hold to $13 \mathrm{~min}$, return to $5 \% \mathrm{~B}$ at $13.01 \mathrm{~min}$ and hold until $14 \mathrm{~min}$. For the MS settings: $2.14 \mathrm{kV}$ capillary voltage, $90^{\circ} \mathrm{C}$ source temperature, $350^{\circ} \mathrm{C}$ desolvation temperature, 600 liters $\mathrm{h}^{-1}$ desolvation nitrogen gas flow rate, $10 \mathrm{~V}$ cone voltage, and $50-1500 \mathrm{~m} / z$ mass range. Three separate acquisition functions were set up to generate spectra at different collision energies $(5,25$, and $60 \mathrm{eV})$.

A Shimadzu LC-20AD HPLC system connected to a Waters LCT Premier ToFMS was used for screening trichome acylsugars of the BILs. Ten microliters trichome metabolites samples were used for reverse-phase separation in a fused core Ascentis Express C18 column $(2.1 \mathrm{~mm} \times 10 \mathrm{~cm}, 2.7 \mu \mathrm{m}$ particle size; SigmaAldrich) with the column temperature of $40^{\circ} \mathrm{C}$. The LC starting conditions were $90 \%$ solvent A ( $0.15 \%$ formic acid in water) and $10 \%$ solvent B (acetonitrile) with a flow rate of $0.4 \mathrm{~mL} / \mathrm{min}$. The 7 -min elution gradient used to separate the trichome metabolites: ramp to $40 \% \mathrm{~B}$ at $1 \mathrm{~min}$, then to $100 \% \mathrm{~B}$ at $5 \mathrm{~min}$, hold at $100 \% \mathrm{~B}$ to $6 \mathrm{~min}$, return to $90 \% \mathrm{~A}$ at $6.01 \mathrm{~min}$ and hold until $7 \mathrm{~min}$. The 40 -min elution gradient used to separate the BIL6180 acylsugars in Supplementary Fig. 1 employed the following linear steps: starting with $95 \%$ solvent $A$ and $5 \%$ solvent $B$, ramp from $5 \% \mathrm{~B}$ to $10 \% \mathrm{~B}$ at $3 \mathrm{~min}$, then to $34 \% \mathrm{~B}$ at $5 \mathrm{~min}$, ramp to $35 \% \mathrm{~B}$ at $20 \mathrm{~min}$, then to $58 \% \mathrm{~B}$ at $21 \mathrm{~min}$, ramp to $59 \% \mathrm{~B}$ at $36 \mathrm{~min}$, then to $100 \% \mathrm{~B}$ at $37 \mathrm{~min}$ and hold to $38 \mathrm{~min}$, return to $5 \% \mathrm{~B}$ at $39 \mathrm{~min}$ and hold until $40 \mathrm{~min}$. For the MS settings: $2.5 \mathrm{kV}$ capillary voltage, $100^{\circ} \mathrm{C}$ source temperature, $350^{\circ} \mathrm{C}$ desolvation temperature, 350 liters $\mathrm{h}^{-1}$ desolvation nitrogen gas flow rate, $10 \mathrm{~V}$ cone voltage, and $50-1500 \mathrm{~m} / z$ mass range. Three separate acquisition functions were set up to generate spectra at different collision energies $(10,40$, and $80 \mathrm{eV})$.

Acylsugar annotations were inferred using collision-induced dissociation $\mathrm{MS}^{25,28}$. The carboxylate fragment ion masses generated by collision-induced dissociation MS in negative ion mode provided information about the number and length of acyl chains. Cleavage of the glycosidic linkage in positive ion mode by collision-induced dissociation MS yields information about acylation patterns of the pyranose and furanose ring of sucrose. Leaf acylsugar abundances were analyzed by the Waters QuanLynx tool to integrate extracted ion peak areas relative to the internal standard peak area, using the data generated in the negativeion mode at the lowest collision energy $(5 \mathrm{eV})$.

Purification of acylsugars for NMR analysis. Thirty BIL6180 plants were used to extract trichome acylsugars for NMR analysis. All plant leaves including the petiole tissues were harvested into a 5 -liter beaker that contained $2 \mathrm{~L}$ of $100 \%$ methanol. After gentle agitation for $2 \mathrm{~min}$, the solvents were transferred into two 1-liter glass bottles and the extraction solvent dried using a rotary evaporator under vacuum and then dissolved in $3 \mathrm{~mL}$ of $80 \%$ acetonitrile with sonication for $10 \mathrm{~min}$. The concentrated solutions were centrifuged at $5000 \times g$ for $10 \mathrm{~min}$ at $25^{\circ} \mathrm{C}$ to remove any insoluble debris and $200 \mu \mathrm{L}$ aliquots were transferred to HPLC vials with glass inserts, and acylsugars were purified as described below.

The S2:10 (iC5, iC5) used for NMR analysis was made through sequential enzymatic reactions. In brief, the first enzymatic step was performed by incubating 
$100 \mu \mathrm{M}$ iC5-CoA, $1 \mathrm{mM}$ sucrose, and Sl-ASAT1 recombinant enzyme in $60 \mathrm{~mL}$ of $50 \mathrm{mM}$ ammonium acetate ( $\mathrm{pH} 6.0$ ) buffer at $30^{\circ} \mathrm{C}$ for $1 \mathrm{~h}$, followed by heat inactivation of the enzyme at $65^{\circ} \mathrm{C}$ for $10 \mathrm{~min}$. The recombinant enzyme SpASAT3 and $100 \mu \mathrm{M}$ of iC5-CoA were then added to the solution for a $1 \mathrm{~h}$ incubation at $30^{\circ} \mathrm{C}$. The reactions were stopped by adding $120 \mathrm{~mL}$ acetonitrile/ isopropanol/formic acid (1:1:0.001), and dried using a vacuum concentrator (SpeedVac ${ }^{\mathrm{TM}}$ ) from Thermo Scientific followed by resuspension in $2 \mathrm{~mL}$ of water with ultrasonication for $10 \mathrm{~min}$. Following centrifugation at $3000 \mathrm{~g}$ for $10 \mathrm{~min}$ at $25^{\circ} \mathrm{C}$, the supernatant was aliquoted into ten glass inserts in HPLC vials.

The acylsugars were purified using a Waters 2795 HPLC system coupled with an LKB BROMMA 221 Superrac fraction collector, using a $200 \mu \mathrm{L}$ injection volume. A Thermo Scientific Acclaim 120 C18 HPLC column $(4.6 \times 150 \mathrm{~mm}, 5 \mu \mathrm{m}$ particle size) was used for all compound separations with the column temperature set at $40^{\circ} \mathrm{C}$. Solvent A ( $0.15 \%$ formic acid in water) and solvent B (acetonitrile) were used as the LC mobile phase with the flow rate of $1.5 \mathrm{~mL} / \mathrm{min}$. The gradient elution methods used to separate the acylsugar compounds are as follows. For separating S3:15 $(5,5,5)$ in BIL6180: $1 \% \mathrm{~B}$ at $0 \mathrm{~min}$ and hold to $2 \mathrm{~min}$, ramp to $10 \%$ $\mathrm{B}$ at $3 \mathrm{~min}$, then to $36 \% \mathrm{~B}$ at $5 \mathrm{~min}$ and hold to $33 \mathrm{~min}$, ramp to $100 \% \mathrm{~B}$ at $34 \mathrm{~min}$ and hold to $38 \mathrm{~min}$, return to $1 \% \mathrm{~B}$ at $39 \mathrm{~min}$ and hold to $40 \mathrm{~min}$. S3:22 $(5,5,12)$ from BIL6180 was purified as follows: $1 \% \mathrm{~B}$ at $0 \mathrm{~min}$ and hold to $2 \mathrm{~min}$, ramp to $10 \% \mathrm{~B}$ at $3 \mathrm{~min}$ and then to $58 \% \mathrm{~B}$ at $5 \mathrm{~min}$, ramp to $60 \% \mathrm{~B}$ at $33 \mathrm{~min}$ and then to $100 \% \mathrm{~B}$ at $34 \mathrm{~min}$, hold $100 \% \mathrm{~B}$ to $38 \mathrm{~min}$, then return to $1 \% \mathrm{~B}$ at $39 \mathrm{~min}$ and hold to $40 \mathrm{~min}$. For separating the enzymatically produced $\mathrm{S} 2: 10$ (iC5,iC5): $1 \% \mathrm{~B}$ at $0 \mathrm{~min}$ and hold to $2 \mathrm{~min}$, ramp to $10 \% \mathrm{~B}$ at $3 \mathrm{~min}$ and then to $14 \% \mathrm{~B}$ at $5 \mathrm{~min}$, ramp to $15 \% \mathrm{~B}$ at $25 \mathrm{~min}$ and then to $100 \% \mathrm{~B}$ at $26 \mathrm{~min}$, hold $100 \% \mathrm{~B}$ to $28 \mathrm{~min}$, then return to $1 \% \mathrm{~B}$ at $29 \mathrm{~min}$ and hold to $30 \mathrm{~min}$

The eluted fractions were collected at a rate of $1 \mathrm{~mL}$ per minute. The fractions containing the desired compounds were verified by LC/MS, pooled and dried under vacuum. NMR spectra were recorded in solutions in Shigemi solventmatched tubes $\left(\mathrm{CDCl}_{3}\right)$ for each purified compound using a Bruker Avance 900 NMR spectrometer equipped with a TCI triple-resonance inverse detection cryoprobe at the Michigan State University Max T. Rogers NMR facility. The ${ }^{1} \mathrm{H}$ spectra were recorded at $899.27 \mathrm{MHz}$, and ${ }^{13} \mathrm{C}$ NMR spectra were recorded at $226.14 \mathrm{MHz}$

The NMR spectra data for S2:10 is as follows: ${ }^{1} \mathrm{H}$ NMR: 5.66 (br., $\left.1 \mathrm{H}\right), 4.87$ (br, $\mathrm{m}, 1 \mathrm{H}), 4.86(\mathrm{br}, \mathrm{m}, 1 \mathrm{H}), 4.33(\mathrm{t}, J=8.3 \mathrm{~Hz}, 1 \mathrm{H}), 4.22(\mathrm{~d}, J=7.5 \mathrm{~Hz}, 1 \mathrm{H}), 4.07(\mathrm{t}$, $J=9.9 \mathrm{~Hz}, 1 \mathrm{H}), 4.01$ (br., $1 \mathrm{H}), 3.82(\mathrm{br}, J=8.1 \mathrm{~Hz}, 1 \mathrm{H}), 3.72,3.89(\mathrm{~m}, J=12.6 \mathrm{~Hz}$, $2 \mathrm{H}), 3.69(\mathrm{~m}, J=13.2 \mathrm{~Hz}, 2 \mathrm{H}), 3.55,3.63(\mathrm{~d}, J=11.5 \mathrm{~Hz}, 2 \mathrm{H}), 2.28(\mathrm{~m}, 2 \mathrm{H}), 2.28$ $(\mathrm{m}, 2 \mathrm{H}), 2.12(\mathrm{~m}, 1 \mathrm{H}), 2.12(\mathrm{~m}, 1 \mathrm{H}), 0.98(\mathrm{br}, 6 \mathrm{H}), 0.98(\mathrm{br}, 6 \mathrm{H}) ;{ }^{13} \mathrm{C}$ NMR: $174.10,173.10,104.3,89.03,81.77,78.66,73.28,72.43,72.30,70.36,69.51,64.32$, $61.21,59.61,43.2,42.27,25.37,25.37,22.14,22.14$; HRMS ( $m / z$, ESI-): calcd. for $[\mathrm{M}+\text { formate }]^{-}=\mathrm{C}_{23} \mathrm{H}_{39} \mathrm{O}_{15}{ }^{-}=555.2294$; found 555.2273 .

The NMR spectra data for S3:15 is as follows: ${ }^{1} \mathrm{H}$ NMR: $5.73(\mathrm{~m}, J=3.6 \mathrm{~Hz}$, $1 \mathrm{H}), 5.52(\mathrm{~m}, J=9.8 \mathrm{~Hz}, 1 \mathrm{H}), 4.91(\mathrm{~m}, J=9.9 \mathrm{~Hz}, 1 \mathrm{H}), 4.85(\mathrm{~m}, J=10.2 \mathrm{~Hz}, 1 \mathrm{H})$, $4.32(\mathrm{t}, J=8.3 \mathrm{~Hz}, 1 \mathrm{H}), 4.26(\mathrm{~d}, J=8.3 \mathrm{~Hz}, 1 \mathrm{H}), 4.25(\mathrm{~m}, 1 \mathrm{H}), 3.77(\mathrm{~m}, 1 \mathrm{H}), 3.75$, $3.86(\mathrm{~d}, J=11.5 \mathrm{~Hz}, 2 \mathrm{H}), 3.64(\mathrm{~m}, J=11.5 \mathrm{~Hz}, 2 \mathrm{H}), 3.49,3.63(\mathrm{~m}, 2 \mathrm{H}), 2.39 \mathrm{c}(\mathrm{m}, J$ $=6.9 \mathrm{~Hz}, 1 \mathrm{H}), 2.37(\mathrm{~m}, J=6.9 \mathrm{~Hz}, 1 \mathrm{H}), 2.30(\mathrm{~m}, J=6.9 \mathrm{~Hz}, 1 \mathrm{H}), 2.22(\mathrm{~d}, J=$ $7.0 \mathrm{~Hz}, 2 \mathrm{H}), 2.20(\mathrm{~d}, J=7.0 \mathrm{~Hz}, 2 \mathrm{H}), 2.12(\mathrm{~d}, J=7.0 \mathrm{~Hz}, 2 \mathrm{H}), 2.05(\mathrm{~m}, 1 \mathrm{H}), 2.05$ $(\mathrm{m}, 1 \mathrm{H}), 2.00(\mathrm{~m}, 1 \mathrm{H}), 1.45,1.67(\mathrm{~m}, 2 \mathrm{H}), 1.43,1.63(\mathrm{~m}, J=7.0 \mathrm{~Hz}, 2 \mathrm{H}), 1.39$, $1.61(\mathrm{~m}, 2 \mathrm{H}), 1.12(\mathrm{~d}, J=6.9 \mathrm{~Hz}, 3 \mathrm{H}), 1.09(\mathrm{~d}, J=6.9 \mathrm{~Hz}, 3 \mathrm{H}), 1.06(\mathrm{~d}, J=6.9 \mathrm{~Hz}$, $3 \mathrm{H}), 0.94(\mathrm{~d}, J=6.7 \mathrm{~Hz}, 6 \mathrm{H}), 0.94(\mathrm{~d}, J=6.7 \mathrm{~Hz}, 6 \mathrm{H}), 0.93(\mathrm{~m}, 3 \mathrm{H}), 0.91(\mathrm{~m}, 3 \mathrm{H})$, $0.91(\mathrm{~d}, J=6.7 \mathrm{~Hz}, 6 \mathrm{H}), 0.90(\mathrm{~m}, 3 \mathrm{H}) ;{ }^{13} \mathrm{C}$ NMR: $176.98,176.07,175.90,173.08$, $172.38,172.32,104.90,89.04,81.64,77.16,73.02,71.30,70.61,69.16,68.55,63.90$, $61.48,60.62,42.95,42.86,42.56,40.74,40.65,40.23,26.33,26.19,26.19,25.18$ $25.13,25.13,22.66,22.26,21.81,16.23,16.20,16.04,11.47,11.42,11.26$; HRMS $(m /$ $z$, ESI-): calcd. for $[\mathrm{M}+\text { formate }]^{-}=\mathrm{C}_{28} \mathrm{H}_{47} \mathrm{O}_{16}{ }^{-}=639.2870$; found 639.2870

The NMR spectra data for S3:22[a] is as follows: ${ }^{1} \mathrm{H}$ NMR: $5.73(\mathrm{~d}, J=3.2 \mathrm{~Hz}$, $1 \mathrm{H}), 5.55(\mathrm{t}, J=9.9 \mathrm{~Hz}, 1 \mathrm{H}), 4.94(\mathrm{~m}, J=10.1 \mathrm{~Hz}, 1 \mathrm{H}), 4.86(\mathrm{dd}, J=3.4,10.2 \mathrm{~Hz}$, $1 \mathrm{H}), 4.30(\mathrm{t}, J=8.3 \mathrm{~Hz}, 1 \mathrm{H}), 4.24(\mathrm{~d}, J=7.9 \mathrm{~Hz}, 1 \mathrm{H}), 4.20(\mathrm{~m}, 1 \mathrm{H}), 3.81(\mathrm{~m}, J=$ $7.4 \mathrm{~Hz}, 1 \mathrm{H}), 3.74,3.89(\mathrm{~d}, J=13.2 \mathrm{~Hz}, 2 \mathrm{H}), 3.64(\mathrm{~m}, J=12.5 \mathrm{~Hz}, 2 \mathrm{H}), 3.53,3.61(\mathrm{~d}$, $J=11.9 \mathrm{~Hz}, 2 \mathrm{H}), 2.40(\mathrm{~m}, J=7.3 \mathrm{~Hz}, 1 \mathrm{H}), 2.37(\mathrm{~m}, J=7.3 \mathrm{~Hz}, 1 \mathrm{H}), 2.22(\mathrm{t}, J=$ $7.1 \mathrm{~Hz}, 2 \mathrm{H}), 1.54(\mathrm{br}, 2 \mathrm{H}), 1.47,1.61(\mathrm{~m}, 2 \mathrm{H}), 1.46,1.69(\mathrm{~m}, 2 \mathrm{H}), 1.30(\mathrm{~m}, 2 \mathrm{H})$, $1.25-1.27(\mathrm{br}, 14 \mathrm{H}), 1.14(\mathrm{~d}, J=6.7 \mathrm{~Hz}, 3 \mathrm{H}), 1.11(\mathrm{~d}, J=6.9 \mathrm{~Hz}, 3 \mathrm{H}), 0.91(\mathrm{~m}$, $3 \mathrm{H}), 0.89$ (m, $3 \mathrm{H}), 0.88$ (m, $3 \mathrm{H}) ;{ }^{13} \mathrm{C}$ NMR: 176.82, 176.14, 172.87, 88.80, 70.90, $68.60,67.90,40.95,40.05,33.75,29.08-31.65,26.53,24.50,22.21,16.30,15.64$ 13.82, 11.20; HRMS ( $/ z / z$, ESI-): calcd. for $[\mathrm{M}+\text { formate }]^{-}=\mathrm{C}_{35} \mathrm{H}_{61} \mathrm{O}_{16}{ }^{-}=$ 737.3965; found 737.3952

The NMR spectra data for S3:22[b] is as follows: ${ }^{1} \mathrm{H}$ NMR: $5.71(\mathrm{~d}, J=3.05 \mathrm{~Hz}$ $1 \mathrm{H}), 4.87(\mathrm{dd}, J=2.72,10.2 \mathrm{~Hz}, 1 \mathrm{H}), 2.40(\mathrm{~m}, J=6.8 \mathrm{~Hz}, 1 \mathrm{H}), 1.13(\mathrm{~d}, J=6.7 \mathrm{~Hz}$, $3 \mathrm{H}), 1.44,1.61(\mathrm{~m}, J=7.2 \mathrm{~Hz}, 2 \mathrm{H}), 0.87(\mathrm{~m}, 3 \mathrm{H}), 5.52(\mathrm{~m}, J=9.7 \mathrm{~Hz}, 1 \mathrm{H}), 2.21$ $(\mathrm{m}, J=7.1 \mathrm{~Hz}, 2 \mathrm{H}), 1.53(\mathrm{br}, 2 \mathrm{H}), 1.27-1.29(\mathrm{br}, 14 \mathrm{H}), 1.29(\mathrm{~m}, J=6.8 \mathrm{~Hz}, 2 \mathrm{H})$, $0.87(\mathrm{~m}, 3 \mathrm{H}), 4.91(\mathrm{~m}, J=9.9 \mathrm{~Hz}, 1 \mathrm{H}), 2.18(\mathrm{~d}, J=7.0 \mathrm{~Hz}, 2 \mathrm{H}), 2.05(\mathrm{~m}, 1 \mathrm{H}), 0.93$ $(\mathrm{m}, J=7.0 \mathrm{~Hz}, 6 \mathrm{H}), 4.23(\mathrm{~m}, 1 \mathrm{H}), 3.60,3.64(\mathrm{~m}, J=12.0 \mathrm{~Hz}, 2 \mathrm{H}), 3.49,3.60(\mathrm{~d}, J=$ $8.7 \mathrm{~Hz}, 2 \mathrm{H}), 4.26(\mathrm{~m}, 1 \mathrm{H}), 4.26(\mathrm{~m}, 1 \mathrm{H}), 3.77(\mathrm{~m}, 1 \mathrm{H}), 3.75,3.87(\mathrm{~d}, J=11.8 \mathrm{~Hz}$, $2 \mathrm{H}) ;{ }^{13} \mathrm{C}$ NMR: $176.92,173.03,172.25,104.5,88.9,81.58,77.82,73.27,71.7,70.78$, $69.16,68.74,64.4,61.7,60.8,42.63,40.30,33.76,29.29-32.05,26.50,25.09,24.52$, $22.35,21.79,15.75,13.86,11.13$; HRMS $\left(\mathrm{m} / z\right.$, ESI-): calcd. for $\left[\mathrm{M}+\right.$ formate $^{-}{ }^{-}=$ $\mathrm{C}_{35} \mathrm{H}_{61} \mathrm{O}_{16}{ }^{-}=737.3965$; found 737.3967 .

Site-Directed mutagenesis. A PCR-based site-directed mutagenesis method was used to introduce mutations to ASATs with the help of Q5 Site-Directed
Mutagenesis Kit (NEB). The presence of mutations was validated by Sanger DNA sequencing of the pET28b plasmids with the genes. The web-based software NEBaseChanger (Version 1.2.2, NEB) was used to design primers for generating mutations, which are listed in Supplementary Table 1.

Protein expression and enzyme assays. Restriction digest cloning was used to clone various ASAT2 or ASAT3 orthologs into the pET28b expression vector. The recombinant proteins were produced using Escherichia coli BL21 Rosetta cells (EMD Millipore) as the host, as described ${ }^{22}$. Nickel affinity chromatography was used to purify the soluble protein ${ }^{26}$

Activity assays for ASAT2 and ASAT3 were performed by incubating purified recombinant enzymes in $30 \mu \mathrm{L}$ of $50 \mathrm{mM}$ ammonium acetate ( $\mathrm{pH}$ 6.0) buffer with $100 \mu \mathrm{M}$ acyl-CoAs. $50 \mu \mathrm{M}$ acylsucrose acceptor-including purified S1:5 (iC5 $\left.{ }^{\mathrm{R} 4}\right)$, $\mathrm{S} 2: 10\left(\mathrm{iC}^{\mathrm{K} 2}, \mathrm{iC} 5^{\mathrm{R} 4}\right)$, and $\mathrm{S} 2: 10\left(\mathrm{iC} 5^{\mathrm{R} 4}\right.$, aiC5 $\left.{ }^{\mathrm{R} 3}\right)$-were used to test $\mathrm{F}$ - and P-type activities of different ASAT2 and ASAT3 orthologs. The assays were kept at $30^{\circ} \mathrm{C}$ for $30 \mathrm{~min}$ and the reactions terminated by addition of $60 \mu \mathrm{L}$ of acetonitrile/ isopropanol/formic acid (1:1:0.001) with $10 \mu \mathrm{M}$ propyl-4-hydroxybenzoate as the internal standard. The reaction solutions were centrifuged at $13,000 \times g$ for $10 \mathrm{~min}$ and the supernatant transferred to glass inserts in HPLC vials for LC/MS analysis. All enzyme reactions were performed at least three times. The representative LC/ MS chromatograms from one of the assays are shown in the figures. The acyl-CoA substrates-iC5-CoA and nC12-CoA-were purchased from Sigma-Aldrich. Chemical synthesis of aiC5-CoA was as described ${ }^{27}$.

\section{ASAT orthologs amplification and phylogenetic analysis. ASAT2 orthologs} cloned from S. lycopersicum through S. corneliomulleri that were used to build the phylogenetic tree in Supplementary Fig. 11 are from a previous study ${ }^{22}$. We amplified additional ASAT2 ortholog sequences from additional accessions of $S$ habrochaites and S. pennellii genomic DNA using the primers listed in Supplementary Table 1 . In the previous report, multiple ASAT3-F and ASAT3-P type alleles were amplified from different accessions of $S$. habrochaites using the F- and $\mathrm{P}$-type primer sets ${ }^{27}$. In the current work, the same primer sets were used to amplify F- and P-type ASAT3 alleles in multiple wild tomato species. CDNA was used as templates to amplify ASAT3 from S. pimpinellifolium, S. galapagense, $S$. neorickii, S. arcanum, S. chilense, S. peruvianum, and S. corneliomulleri. For S. pennellii accessions, genomic DNA was used to amplify ASAT3. The amplified products were cloned into pET28b using the restriction cloning method and the ASAT2 or ASAT3 sequences obtained by Sanger sequencing using plasmids extracted from individual clones. The sequences are in GeneBank with the accession numbers listed below.

For each ASAT2 ortholog, the full coding sequences were used to build the phylogenetic tree. The regions introduced by F- or P- forward primers-24 bp in the $5^{\prime}$ end of the ASAT3 sequences-were eliminated from the phylogenetic analysis to avoid hybrid coding sequences. The nucleotide sequences were aligned using the default MUSCLE algorithm with the MEGA7 software ${ }^{50}$. For both ASAT2 and ASAT3, the Tamura 3-parameter Maximum Likelihood model was chosen for phylogenetic tree construction from 24 different nucleotide substitution models based on the lowest Bayesian Information Criterion. The bootstrap values were obtained after 1000 replicates. The phylogenetic trees in Fig. 4a, Supplementary Figs. 11 and 12 were all made using the above method.

Homology structural modeling of SI-ASAT2 and SI-ASAT3. The Phyre2 web portal $^{51}$ was used to predict the tertiary structures of Sl-ASAT2 and Sl-ASAT3. The Open-Source PyMOL software (Version 1.8.X Schrödinger, LLC) was applied to overlay the Sl-ASAT2 and SI-ASAT3 modeled structures with the trichothecene 3$O$-acetyltransferase structure $(\mathrm{PDB} \text { code } 3 \mathrm{~B} 2 \mathrm{~S})^{38}$

Data availability. The data that support the findings of this study are available from the corresponding author upon reasonable request. The NMR raw data for the four purified compounds S2:10, S3:15, S3:22[a], and S3:22[b] are deposited in the public data repository Open Science Framework under the link https://osf.io/ exfmr/. Sequence data that support the findings of this study are available as GenBank/EMBL data libraries under these accession numbers. ASAT2: Sl-ASAT2 (KT359567), Sp-ASAT2 (KT359557), LA1578-ASAT2 (KT359558), LA1278ASAT2 (KT359559), LA2133-ASAT2 (KT359560), LA1777-ASAT2 (KT359561), LA1401-ASAT2 (KT359562), LA0107-ASAT2 (KT359563), LA1969-ASAT2 (KT359564) LA2172-ASAT2 (KT359565), LA1718-ASAT2 (KT359566), Sl-ASAT3 (KM516150), LA1718-ShASAT2_2 (KY962559), LA1737-ShASAT2_1 (KY962560), LA1737-ShASAT2_2 (KY962561), LA1737-ShASAT2_3 (KY962562), LA1772-ShASAT2 1 (KY962563), LA1772-ShASAT2 2 (KY962564), LA1978 ShASAT2_1 (KY962565), LA1978-ShASAT2_2 (KY962566), LA1978-ShASAT2_3 (KY962567), LA2098-ShASAT2 1 (KY962568), LA2098-ShASAT2_2 (KY962569), LA2156-ShASAT2_1 (KY962570), LA2156-ShASAT2_2 (KY962571), LA2156ShASAT2_3 (KY962572), LA2196-ShASAT2_1 (KY962573), LA2196-ShASAT2_2 (KY962574), LA2650-ShASAT2_1 (KY962575), LA2650-ShASAT2_2 (KY962576), LA2722-ShASAT2_1 (KY962577), LA2722-ShASAT2_2 (KY962578), LA2861 ShASAT2 1 (KY962579), LA2861-ShASAT2 _2 (KY962580), LA2861-ShASAT2 3 (KY962581), LA2975-ShASAT2_1 (KY962582), LA2975-ShASAT2_2 (KY962583), LA2975-ShASAT2_3 (KY962584), LA1282-SpASAT2 (KY962585), LA1356- 
SpASAT2 (KY962586), LA1367-SpASAT2 (KY962587), LA1376-SpASAT2 (KY962588), LA1649-SpASAT2 (KY962589), LA1911-SpASAT2 (KY962590), LA1926-SpASAT2 (KY962591), LA1946-SpASAT2 (KY962592), LA2560SpASAT2_1 (KY962593), LA2560-SpASAT2_2 (KY962594), LA2963-SpASAT2 (KY962595)

ASAT3: Sl-ASAT3 (KM516150), Sp-ASAT3 (KM516151), LA1777-ShASAT3-F (KM516152), LA1978-ShASAT3-F (KM516153), LA2156-ShASAT3-F (KM516154), LA2204-ASAT3-like1 (KM516155), LA2861-ShASAT3-F (KM516156), LA2861-ShASAT3-P (KM516157), LA2156-ASAT3-like (KM516158), LA1777-ShASAT3-P (KM516159), LA1731-ShASAT3-P (KM516160), LA1731-ShASAT3-F (KM516161), LA2722-ShASAT3-P (KM516162), LA2650-ShASAT3-P (KM516163), LA2574-ShASAT3-P (KM516164), LA2204-ASAT3-like2 (KM516165), LA1772-ShASAT3-P (KM516166), LA2098-ShASAT3-P (KM524335), LA1926-SpASAT3 (KY962596), LA2560-SpASAT3 (KY962597), LA1946-SpASAT3 (KY962598), LA1941SpASAT3 (KY962599), LA1911-SpASAT3 (KY962600), LA1649-SpASAT3 (KY962601), LA1376-SpASAT3_2 (KY962602), LA1367-SpASAT3_2 (KY962603), LA1367-SpASAT3_1 (KY962604), LA1356-SpASAT3_2 (KY962605), LA1356SpASAT3_1 (KY962606), LA1282-SpASAT3_3 (KY962607), LA1282-SpASAT3_2 (KY962608), LA1282-SpASAT3_1 (KY962609), LA2963-SpASAT3 (KY962610), LA1376-SpASAT3_1 (KY962611), LA1578-ASAT3_1 (KY962612), LA1578ASAT3_2 (KY962613), LA2133-ASAT3 (KY962614), LA2172-ASAT3_1 (KY962615), LA2172-ASAT3_2 (KY962616), LA1364-ASAT3 (KY962617), LA1401-ASAT3 (KY962618), LA1969-ASAT3 (KY962619), LA1278-ASAT3_1 (KY962620), LA1278-ASAT3_2 (KY962621), LA0107-ASAT3_2 (KY962622), LA0107-ASAT3_1 (KY962623), LA0107-ASAT3_3 (KY962624).

Received: 11 May 2017 Accepted: 3 November 2017

Published online: 12 December 2017

\section{References}

1. Wurtzel, E. T. \& Kutchan, T. M. Plant metabolism, the diverse chemistry set of the future. Science 353, 1232-1236 (2016).

2. Pichersky, E., Noel, J. P. \& Dudareva, N. Biosynthesis of plant volatiles: nature's diversity and ingenuity. Science 311, 808-811 (2006).

3. De Luca, V. \& St-Pierre, B. The cell and developmental biology of alkaloid biosynthesis. Trends. Plant. Sci. 5, 168-173 (2000).

4. Banks, J. A. et al. The Selaginella genome identifies genetic changes associated with the evolution of vascular plants. Science 332, 960-963 (2011).

5. Pichersky, E. \& Lewinsohn, E. Convergent evolution in plant specialized metabolism. Annu. Rev. Plant. Biol. 62, 549-566 (2011).

6. Schilmiller, A. L., Pichersky, E. \& Last, R. L. Taming the hydra of specialized metabolism: How systems biology and comparative approaches are revolutionizing plant biochemistry. Curr. Opin. Plant. Biol. 15, 338-344 (2012).

7. Moghe, G. \& Last, R. L. Something old, something new: conserved enzymes and the evolution of novelty in plant specialized metabolism. Plant. Physiol. 169, 1512-1523 (2015).

8. Milo, R. \& Last, R. L. Achieving diversity in the face of constraints: lessons from metabolism. Science 336, 1663-1667 (2012).

9. Panchy, N., Lehti-Shiu, M. D. \& Shiu, S.-H. Evolution of gene duplication in plants. Plant Physiol. 171, 2294-2316 (2016).

10. Hult, K. \& Berglund, P. Enzyme promiscuity: mechanism and applications. Trends Biotechnol. 25, 231-238 (2007).

11. Khersonsky, O. \& Tawfik, D. S. Enzyme promiscuity: a mechanistic and evolutionary perspective. Annu. Rev. Biochem. 79, 471-505 (2010).

12. Aharoni, A. et al. The 'evolvability' of promiscuous protein functions. Nat. Genet. 37, 73-76 (2005).

13. Weng, J. K. \& Noel, J. P. The remarkable pliability and promiscuity of specialized metabolism. Cold Spring Harb. Symp. Quant. Biol. 77, 309-320 (2012).

14. Luu, V. T. et al. $\mathrm{O}$-acyl sugars protect a wild tobacco from both native fungal pathogens and a specialist herbivore. Plant. Physiol. https://doi.org/10.1104/ pp.16.01904 (2017).

15. Liedl, B. E. et al. Acylsugars of wild tomato Lycopersicon pennellii alters settling and reduces oviposition of Bemisia argentifolii (Homoptera: Aleyrodidae). J. Econ. Entomol. 88, 742-748 (1995).

16. Leckie, B. M. et al. Differential and synergistic functionality of acylsugars in suppressing oviposition by insect herbivores. PLOS ONE 11, 1-19 (2016)

17. Hawthorne, D. J., Shapiro, J. A., Tingey, W. M. \& Mutschler, M. A. Trichomeborne and artificially applied acylsugars of wild tomato deter feeding and oviposition of the leafminer Liriomyza trifolii. Entomol. Exp. Appl. 65, 65-73 (1992).

18. Stork, W. F. J., Weinhold, A. \& Baldwin, I. T. Trichomes as dangerous lollipops: do lizards also use caterpillar body and frass odor to optimize their foraging? Plant Signal. Behav. 6, 1893-1896 (2011).
19. Weinhold, A. \& Baldwin, I. T. Trichome-derived $O$-acyl sugars are a first meal for caterpillars that tags them for predation. Proc. Natl Acad. Sci. USA 108, 7855-7859 (2011).

20. St-Pierre, B. \& Luca, V. De. in Recent Advances in Phytochemistry Vol. 34 (eds. Romeo, J. T., Ibrahim, R., Varin, L. \& Luca, V. De) 285-315 (Elsevier, 2000).

21. D'Auria, J. C. Acyltransferases in plants: a good time to be BAHD. Curr. Opin. Plant. Biol. 9, 331-340 (2006).

22. Fan, P. et al. In vitro reconstruction and analysis of evolutionary variation of the tomato acylsucrose metabolic network. Proc. Natl Acad. Sci. USA 113, E239-E248 (2016).

23. Nadakuduti, S. S., Uebler, J. B., Liu, X., Jones, A. D. \& Barry, C. S. Characterization of trichome-expressed BAHDs in Petunia reveals distinct acylsugar assembly mechanisms. Plant. Physiol. 175, 36-50 (2017).

24. Moghe, G. D., Leong, B. J., Hurney, S., Jones, A. D. \& Last, R. L. Evolutionary routes to biochemical innovation revealed by integrative analysis of a plantdefense related specialized metabolic pathway. eLife 6, e28468 (2017).

25. Fan, P., Moghe, G. D. \& Last, R. L. Comparative biochemistry and in vitro pathway reconstruction as powerful partners in studies of metabolic diversity. Methods Enzymol. 576, 1-17 (2016).

26. Schilmiller, A. L., Charbonneau, A. L. \& Last, R. L. Identification of a BAHD acetyltransferase that produces protective acyl sugars in tomato trichomes. Proc. Natl Acad. Sci. USA 109, 16377-16382 (2012).

27. Schilmiller, A. L. et al. Functionally divergent alleles and duplicated loci encoding an acyltransferase contribute to acylsugar metabolite diversity in Solanum trichomes. Plant Cell. 27, 1002-1017 (2015).

28. Ghosh, B., Westbrook, T. C. \& Jones, A. D. Comparative structural profiling of trichome specialized metabolites in tomato (Solanum lycopersicum) and $S$. habrochaites: acylsugar profiles revealed by UHPLC/MS and NMR. Metabolomics 10, 496-507 (2014).

29. Kim, J. et al. Striking natural diversity in glandular trichome acylsugar composition is shaped by variation at the acyltransferase2 locus in the wild tomato Solanum habrochaites. Plant Physiol. 160, 1854-1870 (2012).

30. Schilmiller, A. L., Gilgallon, K., Ghosh, B., Jones, A. D. \& Last, R. L. Acylsugar acylhydrolases: carboxylesterase-catalyzed hydrolysis of acylsugars in tomato trichomes. Plant Physiol. 170, 1331-1344 (2016).

31. Schilmiller, A. L. et al. Monoterpenes in the glandular trichomes of tomato are synthesized from a neryl diphosphate precursor rather than geranyl diphosphate. Proc. Natl Acad. Sci. USA 106, 10865-10870 (2009).

32. Schilmiller, A. et al. Mass spectrometry screening reveals widespread diversity in trichome specialized metabolites of tomato chromosomal substitution lines. Plant J. 62, 391-403 (2010).

33. Ning, J. et al. A feedback insensitive isopropylmalate synthase affects acylsugar composition in cultivated and wild tomato. Plant Physiol. 160, 1821-1835 (2015).

34. Kim, J. et al. Analysis of natural and induced variation in tomato glandular trichome flavonoids identifies a gene not present in the reference genome. Plant Cell 26, 3272-3285 (2014).

35. Ofner, I., Lashbrooke, J., Pleban, T., Aharoni, A. \& Zamir, D. Solanum pennellii backcross inbred lines (BILs) link small genomic bins with tomato traits. Plant J. 87, 151-160 (2016).

36. Fulop, D. et al. A new advanced backcross tomato population enables high resolution leaf QTL mapping and gene identification. G3 Genes, Genomes, Genet. 6, 3169-3184 (2016)

37. Rodriguez, F., Wu, F., Ané, C., Tanksley, S. \& Spooner, D. M. Do potatoes and tomatoes have a single evolutionary history, and what proportion of the genome supports this history? BMC. Evol. Biol. 9, 191 (2009).

38. Garvey, G. S., McCormick, S. P. \& Rayment, I. Structural and functional characterization of the TRI101 trichothecene 3-O-acetyltransferase from Fusarium sporotrichioides and Fusarium graminearum: kinetic insights to combating Fusarium head blight. J. Biol. Chem. 283, 1660-1669 (2008).

39. Wagner, A. The molecular origins of evolutionary innovations. Trends Genet. 27, 397-410 (2011).

40. Szappanos, B. et al. Adaptive evolution of complex innovations through stepwise metabolic niche expansion. Nat. Commun. 7, 11607 (2016).

41. Lewinsohn, E. \& Gijzen, M. Phytochemical diversity: the sounds of silent metabolism. Plant Sci. 176, 161-169 (2009).

42. Harms, M. J. \& Thornton, J. W. Evolutionary biochemistry: revealing the historical and physical causes of protein properties. Nat. Rev. Genet. 14, 559-571 (2013).

43. Sarkinen, T. et al. A phylogenetic framework for evolutionary study of the nightshades (Solanaceae): a dated 1000-tip tree. BMC Evol. Biol. 13, 214 (2013).

44. Pease, J. B., Haak, D. C., Hahn, M. W. \& Moyle, L. C. Phylogenomics reveals three sources of adaptive variation during a rapid radiation. PLoS Biol. 14, 1-24 (2016).

45. Shapiro, J. A., Steffens, J. C. \& Mutschler, M. A. Acylsugars of the wild tomato Lycopersicon pennellii in relation to geographic distribution of the species. Biochem. Syst. Ecol. 22, 545-561 (1994). 
46. Engler, C., Kandzia, R. \& Marillonnet, S. A one pot, one step, precision cloning method with high throughput capability. PLOS ONE 3, e3647 (2008).

47. Karimi, M., Inze, D. \& Depicker, A. GATEWAY vectors for Agrobacterium-mediated plant transformation. Trends Plant. Sci. 7, 193-195 (2002).

48. Eamens, A. L. \& Waterhouse, P. M. in Plant Chromosome Engineering: Methods and Protocols Vol. 701 (ed. Birchler, J. A.) 179-197 (Humana Press, 2011).

49. McCormick, S. in Plant Tissue Culture Manual (ed. Lindsey, K.) 1-9 (Kluwer Academic Publishers, 1991).

50. Kumar, S., Stecher, G. \& Tamura, K. MEGA7: Molecular evolutionary genetics analysis version 7.0 for bigger datasets. Mol. Biol. Evol. 33, 1870-1874 (2016).

51. Kelley, L. A., Mezulis, S., Yates, C. M., Wass, M. N. \& Sternberg, M. J. E. The Phyre2 web portal for protein modeling, prediction and analysis. Nat. Protoc. 10, 845-858 (2015).

\section{Acknowledgements}

We thank the C.M. Rick Tomato Genetics Resource Center (University of California Davis, CA USA) for providing wild tomato species seeds, Zamir lab in Hebrew University of Jerusalem for providing tomato BILs seeds. We acknowledge members of the Solanum Trichome Project for their contributions to this work: especially Kathleen Imre for her help with tomato transformations; Anthony Schilmiller for providing plasmid constructs; Bryan Leong, Gaurav Moghe, and Daniel Lybrand for their helpful comments on the manuscript. We acknowledge the MSU Center for Advanced Microscopy and RTSF Mass Spectrometry and Metabolomics Core Facilities for their support with LC/MS analysis. This work was funded by National Science Foundation grants IOS-1025636 and IOSPGRP-1546617 to A.D.J. and R.L.L. Abigail Miller was supported by NSF REU grant DBI-1358474 in the summer of 2014 and an American Society of Plant Biologists Summer Undergraduate Research Award during the summer of 2015. A.D.J. acknowledges support from the USDA National Institute of Food and Agriculture, Hatch project MICL-02143.

\section{Author contributions}

P.F. and R.L.L. designed research; P.F. and A.M.M., performed experiments; X.L. performed NMR analyses; P.F., A.M.M., X.L., A.D.J., and R.L.L. analyzed data; P.F. and R.L.L. wrote the manuscript. All authors read and edited the manuscript.

\section{Additional information}

Supplementary Information accompanies this paper at https://doi.org/10.1038/s41467017-02045-7.

Competing interests: The authors declare no competing financial interests.

Reprints and permission information is available online at http://npg.nature.com/ reprintsandpermissions/

Publisher's note: Springer Nature remains neutral with regard to jurisdictional claims in published maps and institutional affiliations.

(c) (i) Open Access This article is licensed under a Creative Commons Attribution 4.0 International License, which permits use, sharing, adaptation, distribution and reproduction in any medium or format, as long as you give appropriate credit to the original author(s) and the source, provide a link to the Creative Commons license, and indicate if changes were made. The images or other third party material in this article are included in the article's Creative Commons license, unless indicated otherwise in a credit line to the material. If material is not included in the article's Creative Commons license and your intended use is not permitted by statutory regulation or exceeds the permitted use, you will need to obtain permission directly from the copyright holder. To view a copy of this license, visit http://creativecommons.org/ licenses/by/4.0/.

(C) The Author(s) 2017 